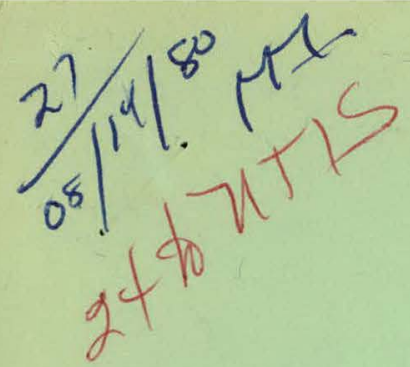

4

\section{E-DIVISION ACTIVITIES REPORT}

Compiled by H. H. Barschall

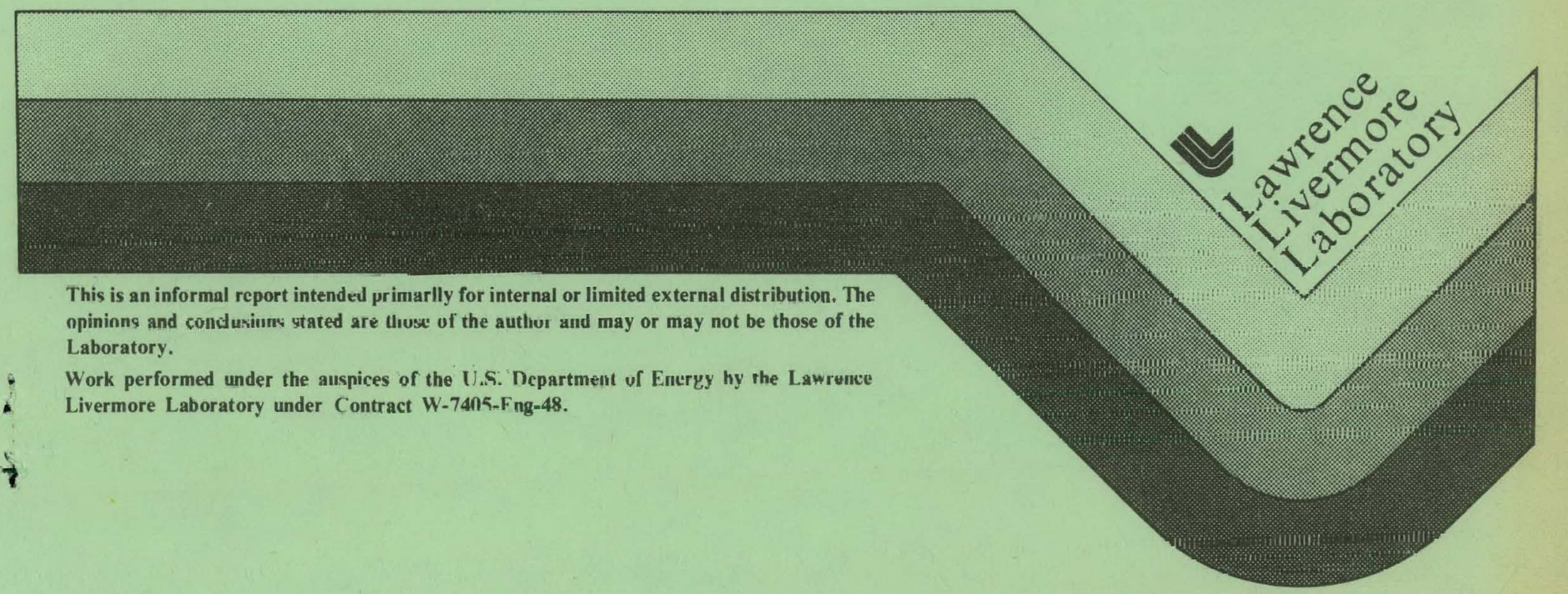




\section{DISCLAIMER}

This report was prepared as an account of work sponsored by an agency of the United States Government. Neither the United States Government nor any agency Thereof, nor any of their employees, makes any warranty, express or implied, or assumes any legal liability or responsibility for the accuracy, completeness, or usefulness of any information, apparatus, product, or process disclosed, or represents that its use would not infringe privately owned rights. Reference herein to any specific commercial product, process, or service by trade name, trademark, manufacturer, or otherwise does not necessarily constitute or imply its endorsement, recommendation, or favoring by the United States Government or any agency thereof. The views and opinions of authors expressed herein do not necessarily state or reflect those of the United States Government or any agency thereof. 


\section{DISCLAIMER}

Portions of this document may be illegible in electronic image products. Images are produced from the best available original document. 
ABSTRACT-1-1 1

INTRODUCTION $-1 .-2$

ACCELERATORS -

Intense Source of 14-MeV Neutrons - 6

EXPERIMENTAL NUCLEAR PHYSICS - 7

Fission - 7

Neutron Cross Sections - 7

Neutron Cross Sections Deduced from Proton Experiments -........... 9

Neutron Radiative Capture -

Photonuclear Reactions - 11

Integral Experiments -

Heavy-Ion Reactions -

Neutron Dosimetry - 13

Electron Scattering -1.-13

THEORETICAL NUCLEAR PHYSICS - 14

Anomalous Optical Potential Parameters for Low Energy Protons -..-. 14

Nuclear Structure Studies --_-1.-15

ELECTRON AND ATOMIC PHYSICS

Ion-Atom Collisions -..- 17

Plasma Spectroscopy - 17

Channeling Radiation - 18

MATERIAL SCIENCE - 20

Experiments - 20

Calculations -1.-1 


\section{CONTENTS (Cont.)}

DEVELOPMENT PROJECTS $\ldots \ldots \ldots$

High Power Microwaves -...............- 22

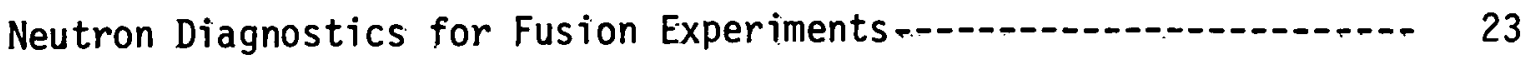

Determination of Atmospheric Oxygen

PUBLICATION LIST - JOURNAL ARTICLES..._. 25

PUBLICATION LIST - CONFERENCE REPORTS

E-DIVISION SCIENTIFIC STAFF _...-...- 30 APPENDICES

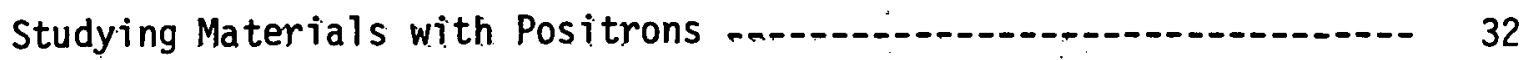

Probing Nuclear Structure with Improved Electron Scattering

Techniques -....... 38

Observation of Channeling Radiation ....-....- 42

Measuring the Hydrogen and Helium Produced by Fusion

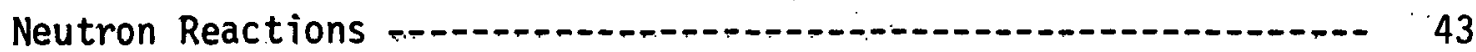


E-Division Activities Report

JuTy 1980

ABSTRACT

This report describes some of the activities in $E$ (Experimental Physics) Division during the past year.

E-Division carries out research and development in areas

related to the missions of the Laboratory. Many of the activities are in pure and applied atomic and nuclear physics. In addition this report describes work on accelerators, radiation damage; microwaves, and plasma diagnostics. 


\section{INTRODUCTION}

E (Experimental Physics) Division carries out basic and applied research in atomic and nuclear physics as well as in other areas related to the missions of the Laboratory. Some of the activities are cooperative efforts with other divisions of the Laboratory, and, in a few cases, with other laboratories. Many of the experiments are directly applicable to problems in weapons and energy, some have only potential applied uses, and others are in pure physics.

E-Division issues an annual report which describes some of the activities in the division during the preceding year. The preceding report (UCID-17271-79) was issued in July 1979. The report gives a brief review of the experimental program of the division. Not all the activities are included every year. The results of the research are published in detail in the scientific literature and are reported at scientific meetings. References to papers and conference proceedings published since the preceding report of this series are listed in the present report.

LLNL publishes monthly in the Energy and Technology Review (UCRL-52000) descriptions of programs of general interest. Articles describing E-Division activities are reprinted in the E-Division annual reports. The following reprints were included in the previous reports:

Probing Nuclei with LLL's Electron Linear Accelerator (1978)

RTNS: A Tool for Studying Neutron Damage (1978)

The Magnetic-Quadrupole Spectrometer: Studying Neutron Reactions (1978)

Pulsed Sphere Measurements for Weapons and Fusion Reactor Design (1978) Measuring Hydrogen-Isotope Distribution Profiles (1978) Nuclear Modeling at the LLL Cyclograaff (1979). 
The present report. includes:

Studying Materials with Positrons

Probing Nuclear Structure with Improved Electron Scattering Techniques Observation of Channeling Radiation

Measuring the Hydrogen and Helium Produced by Fusion Neutron Reactions 


\section{ACCELERATORS}

Most of the activities of the division are centered around seven accelerators: A 100-MeV electron linear accelerator (Linac), three 400-kV dc high-current accelerators, a 3-MV electrostatic accelerator, a 6-MV tandem electrostatic accelerator, and a $76-\mathrm{cm}$ cyclotron. The cyclotron can be used as an injector for the tandem accelerator (cyclograaff). With these accelerators, beams of neutrons, photons, and charged particles can be produced, and the interaction of these projectiles with nuclei, atoms, and solids is studied.

The oldest of the three $400-\mathrm{kV}$ accelerators ("ICT") produces $25 \mathrm{~mA}$ of atomic hydrogen, the newer ones ("RTNS II") are designed to produce $150 \mathrm{~mA}$.

Although all the accelerators can serve as neutron sources, most of the neutron experiments use either the Linac or the 400-kV accelerators. The Linac permits the simultaneous study of neutrons of energies from thermal to about $60 \mathrm{MeV}$ by observation of the neutron time-of-flight over distances as long as $250 \mathrm{~m}$. The $400-\mathrm{kV}$ accelerators serve primarily as intense sources of 14-MeV neutrons.

Most of the charged-particle-interaction experiments are performed with the cyclograaff. Reactions producing charged particles are studied with the aid of a high resolution broad-range magnetic spectrometer as well as with solid-state detection systems. Low-energy gamma rays produced in charged-particle interactions are detected and their energy is measured in a Ge-Li detector, higher-energy gamma rays in a $\mathrm{NaI}$ scintillator with an anticoincidence shield. This latter detector has an unusually large volume: $10 \mathrm{dm}^{3}$ for the $\mathrm{NaI}$ crystal and $1 \mathrm{~m}^{3}$ for the plastic 
scintillator that provides the anticoincidence signal. Neutrons produced in charged-particle reactions are detected and their energy is measured in a well-shielded time-of-flight facility that permits simultaneous observation at 16 angles.

The 3-MV electrostatic accelerator is used for studies of ion-atom collisions and ion interactions with solids.

During the past year efforts were made to improve the performance of the Linac. A new buncher-prebuncher system improves the primary-beam energy resolution in steady-state operation. For improved resolution in neutron cross section measurements and photoneutron experiments using neutron-time-of-flight techniques, an improved gun pulser has been developed. The new pulser permits the production of 2-nsec beam pulses and improves the performance in the 5-10 nsec pulse-width region previously available.

The January 1980 earthquake disturbed the alignment of both the tandem and Linac resulting in a one-month interruption of operation of both accelerators.

The tandem electrostatic accelerator, the cyclotron and the oldest 400-kV high current accelerator share the same operating crew so that there are limitations on their simultaneous operation. During the past year the tandem accelerator was operated by itself for about 500 hours and in combination with the cyclotron for another 1200 hours. Beams produced were protons $80 \%$ of the time, deuterons $7 \%$, and $\mathrm{He}$ and heavier ions the remainder of the time. The ICT accelerator operated for 660 hours. The electron linac served as a neutron source for 1300 hours, as a $\gamma$-ray source for 600 hours, and for other purposes 800 hours. 
Intense Source of 14-MeV Neutrons

The Rotating Target Neutron Source-II (RTNS-II) is a national facility operated for the Fusion Materials Program of the Office of Fusion Energy. Its purpose is to study the effects of fusion neutrons on materials used in the fusion energy program.

During the past year the first of two neutron sources was operated on a regular 80 hour/week schedule. The other source was brought into operation as an ion source test stand. Typically the neutron source operated at a strength of $10^{13} / \mathrm{sec}$; the maximum source strength that has been attained is $2.2 \times 1013 / \mathrm{sec}$. Operation at higher source strengths awaits fabrication of targets of larger diameter than the present $23 \mathrm{~cm}$.

Over 2000 samples from twelve different laboratories were irradiated. Samples of pure metals as well as alloys were irradiated for studies of microstructure and microhardness. Irradiation of ceramic insulators used in TFTR (Tokamak Fusion Test Reactor) served to observe the effect of fusion neutrons on their thermal and mechanical properties. An in-situ creep experiment on $\mathrm{Nb}$ using elevated temperatures of $400-600^{\circ}$ was carried out. Irradiations were performed on low-temperature samples $(4 \mathrm{~K})$ of $\mathrm{NbTi}, \mathrm{Cu}$, and Al for critical current measurements and resistivity studies. The effect of 14-MeV neutrons on the optical properties of quartz and sapphire was studied for the FMIT (Fusion Material Irradiation Test) Facility. Short irradiations were completed on samples of liver enzymes, fiber optic cables, and semiconductor wafers. 


\section{Fission}

The nuclides ${ }^{245} \mathrm{Cm}$ and ${ }^{242} \mathrm{Am}^{\mathrm{m}}$ are two of the major transplutonic elements of concern to such applied problems as actinide burn-up and ${ }^{252} \mathrm{Cf}$ production. Important parameters for determining the reactivity of these fissionable materials are their fission cross sections and the average numbers of neutrons per fission $(\bar{v})$.

Evaluation of previously reported measurements of the fission cross sections of ${ }^{245} \mathrm{Cm}$ and ${ }^{242} \mathrm{Am}^{\mathrm{m}}$ for neutron energies between $1 \mathrm{meV}$ and $20 \mathrm{MeV}$ were completed. The final value of the fission cross section of ${ }^{245} \mathrm{Cm}$ for $2200 \mathrm{~m} / \mathrm{s}$ neutrons is $2080 \pm 40 \mathrm{~b}$. The energy dependence of the fission cross section was analyzed up to a neutron energy of $30 \mathrm{eV}$ with the aid of a multichannel R-matrix code. The analys is yielded parameters for the resonances with an average fission width of 550 meV and a strength function $(\bar{\Gamma} / \bar{D})$ of $1.02 \times 10^{-4}$ consistent with values for neighboring nuclides. A measurement of $\bar{v}$ for ${ }^{245} \mathrm{Cm}$ for thermal neutrons was completed with an accuracy of $2 \%$. The measured value is below previously published values, but above the value obtained by extrapolating previous measurements at higher neutron energy assuming the same energy dependence of $\bar{v}$ as in neighboring nuclides.

A large-area $\left(600 \mathrm{~cm}^{2}\right)$ position-sensitive fission-fragment detector has been developed. It is insensitive to gamma rays. Its time resolution of $\sim 10 \mathrm{nsec}$ permits high enough counting rates so that a rough image of the incident neutron beam from the Linac could be obtained in $20 \mathrm{sec}$.

\section{Neutron Cross Sections}

Cross sections for the production of charged particles in reactions induced by 14-MeV neutrons are of importance to the design of fusion reactors. Previous measurements have investigated structural materials 
(e.g. $\mathrm{Fe}, \mathrm{Cr}, \mathrm{Ni}, \mathrm{Cu}, \mathrm{Mo}$ ) in order to estimate neutron-induced radiation damage. During the past year lighter materials have been studied: ${ }^{2} i$ because of $i$ ts role in tritium breeding, ${ }^{12} \mathrm{C}$ because of $i$ ts potential use as protective curtain for the first wall, ${ }^{16} 0$ and ${ }^{28} \mathrm{Si}$ because of their use in electrical insulators. Some modification of the experimental equipment was required, because these targets have lower $Z$ and hence lower Coulomb barriers than any previously studied, resulting in charged-particlespectra that extend to lower energies than. is the case for heavier nuclei. In particular, the alpha-particle production cross section for ${ }^{12} \mathrm{C}$ and ${ }^{16} 0$ proceeds largely through many-particle-breakup channels $(n+3 \alpha$ and $n+4 \alpha$, respectively) which have alpha spectra extending to below $1 \mathrm{MeV}$. Similarly, the $(n, n \alpha t)$ reaction on ${ }^{7} L i$ yields tritons and alpha particles of very low energy. Use of a thin-window proportional counter for a $\Delta E$ counter allowed alpha particles with energies as low as $0.8 \mathrm{MeV}$ to be detected in a three-element counter-telescope with the quadrupole spectrometer.

The neutron total cross section for ${ }^{3} \mathrm{H}$ from $50 \mathrm{keV}$ to $80 \mathrm{MeV}$ has been measured at the Linac with an accuracy of $0.5 \%$ over most of this energy range. This measurement permits the determination of the zeroenergy cross section, and hence of the singlet, triplet, and coherent $n-T$ scattering lengths, and provides information about the relative strengths of the singlet and triplet interactions. The results disagree with previous measurements.

Previously measured total cross sections of nuclides in the mass -140 region for neutrons of energies between 2.5 and $60 \mathrm{MeV}$ have been analyzed in terms of an optical model potential. The validity of the potential can be checked by calculating angular distribution of elastic scattering and comparing the calculations with available measurements. Agreement between calculations and measurements was obtained for ${ }^{138} \mathrm{Ba}$ and ${ }^{142} \mathrm{Nd}$. 
Potentials deduced by others from proton measurements do not fit the present total cross section measurements we 11.

Neutron Cross Sections Deduced from Proton Experiments

Neutron data for research applications dealing with reactors, material damage studies, shielding calculations, etc. are of ten obtained from optical model (OM) calculations, because measurements of neutron cross sections for many elements are not available. The parameters used in the OM potentials are obtained mainly from "global" sets (smooth dependence on mass number, energy, and neutron excess). These OM parameters predict the trend of the neutron cross sections reasonably well, but when the calculations are compared with measurements, for a given $A$ and a given neutron energy, the values of the parameters need to be optimized to improve the agreement.

According to the Lane-model: of the nucleon-nucleus OM potential, measurements of proton scattering and charge exchange $(p, n)$ reactions to isobaric analog states (IAS) at the appropriate energy may be used to deduce neutron OM parameters. This approach has proved very successful for light and intermediate nuclei, however, multi-step processes proceeding through excited states of the target and excited analog states have a pronounced effect on the ground-state transition and must be understood, if the Lane model is to be applied successfully.

These multi-step processes have been studied previously for isotopes of Mo and Sm for which coupled-channel calculations reproduced the measured charge-exchange cross sections to excited analog states using only parameters derived from proton inelastic scattering and results of the ground-state analog measurements. In view of the good agreement between the experiments and calculations for the Mo and Sm isotopes, measurements of $(p, n)$ charge-exchange cross sections should yield neutron elastic and 
inelastic scattering cross sections. For this purpose measurements on the isotopes $76,80,82$ Se at proton energies of 19, 22, and $25 \mathrm{MeV}$ have been performed. In this experiment the isobaric analogs of the ground states of the target nuclei were observed as well as the one- and twophonon excited analogs.

Because of our interest in the heaviest nuclides an attempt has been made to use the same approach for heavy elements. For this purpose measurements have been performed of the $(p, n)$ IAS cross sections of Ta, $\mathrm{Au}, \mathrm{Bi}, \mathrm{Th}$, and $\mathrm{U}$, and of elastic and inelastic scattering by Th and $\mathrm{U}$ of protons of energies near $26 \mathrm{MeV}$. From these data OM parameters were deduced with a coupled-channel calculation.

Neutron elastic differential cross sections of ${ }^{232}$ Th and ${ }^{238} \mathrm{U}$ at $7 \mathrm{MeV}$ calculated with this potential agree well with measurements. The results confirm that the charge independence of the nuclear optical potential postulated by Lane holds also at these higher $Z$ values and that coupling effects are important for most nuclides.

A knowledge of the number and energy of both alpha particles and secondary neutrons produced in the interaction of $14-\mathrm{MeV}$ neutrons is important for the design of fusion reactors. In addition to the direct measurements of the production cross sections, an effort is underway to deduce the cross sections from proton-induced reactions. The analys is must include precompound processes, and several precompound decay models are being compared with the measurements. Recent measurements have been performed with 26-MeV protons and have involved the following reactions: $(p, n)$ reactions on ${ }^{64} \mathrm{Zn},{ }^{65} \mathrm{Cu}$, and ${ }^{89} \mathrm{Y}$, and $(p, \alpha)$ reactions on ${ }^{89} \mathrm{Y}$ and $209_{B i}$. 
For measurements on nuclear explosions using radiochemical methods a knowledge of the neutron capture cross sections of unstable nuclides is needed. The mass region around $A=90$ is of particular importance: An important parameter in calculations of the cross sections is the $\gamma$-ray strength function. To obtain information on this quantity, the shape of the neutron capture $\gamma$-ray spectrum has been measured using a 3 -crystal $\mathrm{Ge}(\mathrm{L} i)$ spectrometer for the reaction ${ }^{87} \operatorname{Sr}(n, \gamma)$ for thermal neutrons, at $2 \mathrm{keV}$ and $24 \mathrm{keV}$, with neutrons from the Brookhaven High Flux Beam Reactor. The gamma rays from the ${ }^{87} \mathrm{Sr}(n, \gamma)$ reaction were also studied at the Linac for neutron energies between $2 \mathrm{eV}$ and $600 \mathrm{keV}$ with a liquid-scintillator detector. This detector has much poorer $\gamma$-ray energy resolution, but much higher detection efficiency than the $\mathrm{Ge}(L i)$ detector.

The radiative neutron capture by ${ }^{159} \mathrm{~Tb}$ was measured at the Linac as a function of neutron energy to explore correlations between gammaray widths and neutron widths, correlations for which evidence had been found in earlier studies of this reaction.

\section{Photonuclear Reactions}

Photonuclear measurements have been completed at the Linac for a number of nuclides for the energy region from threshold to $30 \mathrm{MeV}$ with monoenergetic photons from positron annihilation in flight. For ${ }^{3} \mathrm{H}$ and

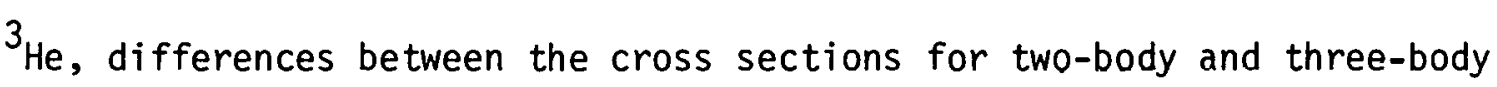
breakup modes compensate in their sum, so that the total photon absorption cross sections for ${ }^{3} \mathrm{H}$ and ${ }^{3} \mathrm{He}$ are nearly the same in both size and shape at energies near and above their peaks.

Recently measured photoneutron cross sections of ${ }^{4} \mathrm{He}$ agree with an older measurement and are about half the photoproton cross sections. The shape of the energy dependence of ${ }^{17} 0$ cross section was studied in 
detail. The photoneutron and photofission cross sections of $235,236,238_{U}$ and ${ }^{232}$ Th have been measured, and the photofission prompt-neutron multiplicities have been determined.

\section{Integral Experiments}

In last year's report (UCID-17223-79) results of measurements of lowenergy neutron spectra emitted by a sphere of $\sigma_{L i D}$ were reported. In these measurements a source of $14-\mathrm{MeV}$ neutrons was at the center of the sphere, and the neutrons were observed with a ${ }^{6} \mathrm{Li}$ glass detector. The earlier report stated that there was good agreement between calculated and observed neutron spectra above $27 \mathrm{keV}$ neutron energy. This agreement now appears to have been fortuitous.

To check the efficiency of the ${ }^{6} \mathrm{Li}$ glass detector the low-energy neutron emission spectrum from a $\mathrm{CH}_{2}$ sphere was measured. This spectrum can be calculated reliably. The measurement therefore provides a calibration of the detector efficiency. With the new efficiency there appears to be a $25 \%$ difference between the calculated and measured spectrum from the ${ }^{6}$ LiD sphere, between $27 \mathrm{KeV}$ and $1.4 \mathrm{MeV}$. The reason for this difference is not yet understood.

\section{Heavy-Ion Reactions}

De-excitation products in the reaction ${ }^{40} \mathrm{Ca}\left({ }^{16} 0, \mathrm{x}\right)$ were measured at the LBL 88" cyclotron at bombarding energies of 104 and $214 \mathrm{MeV}$. Two-body fission-like coincidence yields and light particle spectra have been measured both in singles and in coincidence with evaporation residue fragments. The results have been analyzed using a new approach to the Hauser-Feshbach theory which takes deformation into account in the calculation of transmission coefficients. The observed fissionlike yield $(\leq 6 \mathrm{mb})$ agrees with the new approach $(10 \mathrm{mb})$, but not with 
the old one $(200 \mathrm{mb})$. In addition, when compared to calculations in which the deformation effect on transmission coefficients is ignored, the new approach predicts a substantial increase in the number of high-energy $\alpha$ particles produced (" $\alpha$ decay'amplification"). The $\alpha$-particle spectra observed in this experiment are in good agreement with this new prediction. Neutron Dosimetry

Dose is defined as energy absorbed per unit mass. The only direct method for measuring neutron dose is a determination of the temperature rise caused by the neutrons. Since in most available neutron fluxes this temperature rise is very small. and difficult to measure, most neutron dose measurements deduce dose from a measurement of the ionization produced by the neutrons or from a measurement of the neutron fluence. The conversion from ionization or fluence to dose involves quantities that are not well known.

In cooperation with the Memorial Sloan-Kettering Cancer Center the three dosimetric techniques were compared for $15-\mathrm{MeV}$ neutrons. The results obtained by the three methods agreed more closely than the estimated uncertainties so that the indirect dosimetric methods appear to be very reliable. Electron Scattering

Electron scattering probes fundamental properties of nuclei, such as the charge and magnetization distributions of nuclear ground states and the transition charge densities between the ground and excited states. Collaborative experiments with MIT have been performed at Bates on the rare isotopes of oxygen, and silicon.

Results include delineation of the charge distributions of the oxygen isotopes, determination of the magnetization distribution of 170 , information about the form factors and transition charge densities of excited states of 180, and a comparison of electron- and proton-scattering form factors. 
Anomalous Optical Potential Parameters for Low Energy Protons

Optical model potentials are needed for both direct- and compoundnuclear reaction calculations. The elastic scattering cross sections for charged particles at or below the Coulomb barrier have not been studied as extensively as those above the barrier, because at the lower energies the scattering is nearly that due to a charged sphere. Recently, measurements of the absorption and elastic scattering cross sections for lowenergy protons on targets with mass number about 100 have been found to be inconsistent with conventional optical model parameters based on higher-energy measurements. A modulation of the imaginary potential with mass number was required to fit the data. Especialty large values for this potential were needed for targets between closed shells.

Two possible explanations for this behavior are that 1) the number of two-particle one-hole states for nuclei between closed shells is larger than for nuclei at or near closed shells or 2) coupling effects to specific collective states increase the absorption probability. The two-particle one-hole states would be the states into which the single particle states would decay as the first stage of compound nucleus formation; thus, an enhancement in the number of these states might yield a larger absorption cross section.

Calculations of the two-particle one-hole state densities and of the coupling effects due to collective states have been performed to examine the relative importance of these two effects on the reaction cross section. Both the modulation of the two-particle one-hole state derisity with mass number $(A)$ and the coupling effects are found to produce enhancements in the proton absorption cross section below the Coulomb barrier for nuclides 
between closed shells. Neither of these is as important above the Coulomb barrier, which explains why previous analyses above the Coulomb barrier did not find evidence for shell effects.

These calculations provide support for the recent measurements and original optical model analysis. Initially, many felt that either the measurement or the original analys is was wrong. Because of level density modulations and coupled-channel effects, absorption cross sections for. protons below the Coulomb barrier show significant shell effects. In extreme cases, changes as large as 50\% in low energy transmission coefficients can be produced.

\section{Nuclear Structure Studies}

The nuclear weapons program often makes use of neutron cross sections of unstable nuclides. Because it is difficult or impossible to measure these cross sections, calculations based on appropriate nuclear models are used instead. For cases where the Hauser-Feshbach compound statistical model applies, nuclear level densities are required. In order to calculate realietic nuclear level densities and slrenyth functions for unstable nuclides we have applied the spectral distribution theory so that the effect of two-body interactions may be included. (Except for pairing, such effects cannot be included in a Fermi gas calculation). This theory uses various moments of the two-body hamiltonian (and other operators) which are calculated in a large single-particle basis using the previously developed Vector Model Code. This code has now been enlarged so that it can be applied to large single-particle basis nuclei, e.g. for $A \gtrsim 60$. It is now possible to make calculations for nuclei, such as $U$ or heavier.

In order to calculate hamiltonian moments larger than the second moment in these large spaces, a Monte Carlo method has been developed 
to generate random shell-model vectors. An example of a calculation in which this technique was used was for the ${ }^{28}$ Si level density. The shell model basis used, 12 nucleons in the $d_{5 / 2}, s_{1 / 2}, d_{3 / 2}$ and $f_{7 / 2}$ shells, has a dimension of $5.5 \times 10^{7}$ and at the present time such a large basis would be impossible to treat using exact diagonalization techniques. 
Ion-Atom Collisions

The primary goal of this effort is to understand the mechanisms that produces inner-shell vacancies in ion-atom collisions. Auger-electron measurements have been performed using various light projectiles on gaseous and solid targets (thin foils). The data have been analyzed using the quasi-molecule formed during the collision for the interpretation of lowvelocity collisions. The data indicate anomalously large cross sections for collisional loss of inner shell vacancies. It is expected that simple two-level models may be used for this analysis, since the innershell energy levels are relatively isolated from other levels to which they may couple. Existing analytical models for two-level coupling have been improved by inclusion of translation factors (i.e., taking into account the fact that the active electronic states in the two atoms move relative to one another). Calculations are also being performed with Hartree-Fock wave functions for the quasi-molecule in order to test if simple analytical models give more realistic estimates of the transition probabilities.

\section{Plasma Spectroscopy}

Plasma spectroscopy is a new activity in the Atomic Physics group. Its aim is to produce a well characterized plasma and to study atomic collision phenomena that are ordinarily inaccessible in the laboratory. This information is of interest to the study of plasmas produced by lasers, to magnetically confined plasmas, and to relativistic electron beam machines. 
An experimental plasma light source using a Z-pinch is under construction. This device should permit measurements of the properties of plasmas by observation of ultraviolet and $x$-radiation. Information that is expected to be obtained from the device includes spectroscopic determination of line shift and line broadening, radiation transport, and measurement of collisional excitation rates. The observations should test the ability of available codes to calculate relevant cross sections.

While the Z-pinch machine is being constructed, experiments are being performed on existing plasma devices. Spectra have been observed at the TMX experiment in the magnetic fusion division. Grazing incidence measurements identified impurities and showed the importance of radiation losses caused by $\mathrm{Ti}$. With a normal incidence spectrometer ion temperatures as low as $10 \mathrm{eV}$ and field strengths of $40 \mathrm{~V} / \mathrm{cm}$ could be measured.

A spectrometer has been installed on the target chamber of the Argus laser. Spectra from gold disk targets showed emission lines and absorption edges superimposed on a continuum.

At Physics International high-resolution grazing-incidence spectra were obtained on the relativistic electron beam machine. The spectra show both lines and a continuum and yield information about radiation transport.

\section{Channeling Radiation}

Relativistic charged particles channeled in crystals, either along an axis or between planes, traverse periodic trajectories. This motion results in forward-directed emission of electromagnetic waves which, for MeV electrons, has spectral peaks in the $x$-ray or $\gamma$-ray portion of the spectrum. 
In collaboration with Stanford experiments have been performed at the Linac to study the radiation both from electrons and positrons channeled in thin Si crystals. With electrons and positrons of energies in the tens of MeV range the forward directed photons of energies between 20 and $130 \mathrm{keV}$ were studied. Planar-channeled positrons were observed to give a single spectral peak in the emission. Radiation from planar-channeled electrons occurred in as many as five spectral peaks. The energy dependence of the channeling radiation varies as $\gamma^{1.5}$ to $\gamma^{2}$, where $\gamma$ is the ratio of particle energy to rest energy. At the spectral peak, the measured channeling radiation was several times more intense than bremsstrahiung. Planar-channeling emission linewidths ranged form $10-25 \%$ of the photon energy. From the observed photon energies the interplanar potential in the crystal was determined with a resolution of $\simeq 0.7 \AA$. 


\section{MATERIAL SCIENCE}

\section{Experiments}

One of the most difficult problems in the design of a fusion reactor is the short lifetime of the wall of the plasma-containing vessel caused by radiation damage induced by $14-\mathrm{MeV}$ neutrons. The selection of a suitable material requires a better understanding of the factors that influence the behavior of materials under bombardment by $14-\mathrm{MeV}$ neutrons. Because of the low intensity of available 14-MeV neutron sources, other high-energy neutron sources are often used in radiation damage studies, or protons or other charged particles serve to simulate the effects of neutrons. In experiments us.ing either neutrons or protons, radiation damage may be detected by measuring the lifetime of positrons introduced in the damaged area or by measuring the doppler broadened energy distribution of the annihilation gamma ray. While the positron life-time is sensitive to the electron density, the energy distribution yields information about the electron momentum distribution. Both techniques are sensitive to changes in positron annihilation resulting from defect structure introduced by radiation damage.

In addition to an effective threshold in the energy of recoiling atoms observed in the production of positron traps in irradiations of copper and silver with protons and neutrons, radiation annealing of the damage produced in silver was observed. The radiation annealing volume was observed to vary, growing smaller at high doses at which there is a high probability of overlapping damage sites. The dependence of the annealing volume on recoil spectrum provides additional evidence in support of the existence of a recoil energy threshold. 


\section{Calculations}

A comparison of radiation damage experiments performed with different types of radiation requires calculations of the damage production cross section and damage energy as a function of recoil energy for the different types of radiation. Such calculations for charged particles or high-energy neutrons require a knowledge of the cross sections for the several types of reaction processes. For these calculations a nuclear physics code that calculates reaction cross sections for light reaction products has been modified, by including a calculation of the heavy-recoil energy distribution. Also an optical model calculation of the energy distribution of recoils from elastic scattering has been added. This code permits calculation of the elastic and inelastic damage energy produced by deuterons, alpha particles and heavier ions. The code makes it possible to calculate damage energy for neutrons of energies above those at which relevant cross sections have been measured. The calculation of damage energy from protons includes the cross sections for the production of hydrogen and helium isotopes and other light ions. The presence of hydrogen and helium has a pronounced effect on the final damage configuration in recovery and void formation. 


\section{DEVELOPMENT PROJECTS}

\section{High Power Microwaves}

High-intensity electromagnetic fields in the microwave frequency range are useful for heating plasmas, for generating intense chargedparticle beams, and other applications. A method is under development for filling a superconducting resonant cavity with rf energy for a relatively long time and then extracting the energy during a much shorter time to achieve a power gain.

Interference-type fast microwave switches have bene applied to power levels more than an order of magnitude greater than previously. Using a room-temperature (copper) S-band cavity and a switch built by the Research Advisory Institute, a power gain of approximately 100 was achieved ( $2 \mu \mathrm{sec}$ input pulse, 10 nsec output). The drive power was provided by a klystron and modulator of the Linac. Switching was done by an electron beam fired through the switch cavity by a Marx-bank-Blumlein-diode arrangement. Output pulses of about $30 \mathrm{MW}$ peak power were achieved at $300 \mathrm{~kW}$ drive power.

At higher drive power, about $10 \mathrm{~J}$ of energy was stured In lhe cdvily. This should yield a $10 \mathrm{nsec}$ GW pulse. Adequate energy in the Marx bank was not available at the time to switch out the energy at such high power levels. The intense fields (several MV across the switch waveguide) probably caused the switching beam to blow up before switchout could occur. At the higher power levels, intense high-energy $x$-rays emanated from the cavity walls in an intensity pattern corresponding to a standing microwave. The plan is to repeat the experiment at higher power levels with a larger Marx bank and improved $x$-ray shielding. 
In collaboration with the California Institute of Technology the rf superconducting properties of thin films $(0.1$ to $3 \mu \mathrm{m})$ of superconductors on $\mathrm{Cu}$ substrates have been studied. Films of $\mathrm{Nb}$ on $\mathrm{Cu}$ were prepared by both vapor deposition and sputtering. Excellent surface resistances were obtained, in some cases as good as in bulk Nb at $4.2 \mathrm{~K}$. The thermal stability provided by the $\mathrm{Cu}$ substrate appears to inhibit thermomagnetic breakdown. In addition to having greater thermal stability, superconducting $r f$ cavities are easier to make out of $\mathrm{Cu}$ coated with $\mathrm{Nb}$ than out of bulk Nb. All the thin film cavities studied so far, have been assembled in air, and, unlike cavities of solid $\mathrm{Nb}$, they do not appear to require elaborate surface treatment.

Other thin-film superconductors have been tested in rf cavities, but have not performed as well as the $\mathrm{Nb}$ film on $\mathrm{Cu}$. Surface analyses of the films are being carried out to determine if there are correlations between the measured $r f$ surface resistances and oxides and/or other impurities in the surface layers of the materials.

\section{Neutron Diagnostics for Fusion Experiments}

Measurement of important plasma parameters in fusion experiments is made difficult by high densities and large volumes which prevent the escape of atoms and ions from the central regions, but neutrons suffer negligible attenuation in the plasmas. Knowledge of the neutron generation rate in the plasma may serve to determine primary plasma parameters, such as ion temperature, ion energy confinement time, ion density, spatial and temporal distributions of density and temperature, and may provide data for deducing the plasma power balance. The diagnostics is being developed in cooperation with the Princeton fusion group. A neutron spectrometer system has been built which has an energy resolution of $160 \mathrm{keV}$ at $2.5 \mathrm{MeV}$ and $550 \mathrm{keV}$ 
at $14 \mathrm{MeV}$. With this system measurements have been carried out during time windows as narrow as $1 \mathrm{sec}$ and will soon be extended to $10 \mathrm{msec}$ at detector count rates up to $7 \times 10^{6} \mathrm{sec}^{-1}$. The electronics including the pulse height analyser attaches to the base of a photomultiplier tube within the collimator-shield. Four such systems within a shield will view different plasma regions.

A preliminary version of the system has served to relate neutron yield and spectral data on a TMX end plug to plasma line density, injected neutral beam current, and plasma diamagnetism. These measurements allowed a determination of the ion mean energy as a function of time, of the ion energy confinement time, and of the total fusion power. Determination of Atmospheric Oxygen

In collaboration with the Lawrence Berkeley Laboratory a laser experiment has been begun to measure the variations of the $0_{2}$ concentration in the atmosphere as a function of time for the past 20 years. The purpose of the experiment is to determine the ratio of the change in $0_{2}$ concentration to $\mathrm{CO}_{2}$ concentration to elucidate the location of the $\mathrm{CO}_{2}$ from fossil-fuel burmirig. Only about half the $\mathrm{r}_{2} \mathrm{n}_{2}$ is accounted for by the Scripps Institute measurements of the last twenty years on the $\mathrm{CO}_{2}$ variations in the atmosphere. The present experiment uses precision measurements of the Raman scattering intensity from $\mathrm{N}_{2}$ and $\mathrm{O}_{2}$ in air samples gathered at locations ranging from the equator to the poles. The experiment requires initially a precision of $\approx 3 \times 10^{-6}$. Su far a stability of $2 \times 10^{-6}$ has been achieved for Rayleigh scattering from air. The signal-to-noise ratios of Raman scattering in $\mathrm{O}_{2}$, $\mathrm{N}_{2}$, and $\mathrm{CO}_{2}$ are under study. 


\section{PUBLICATION LIST - .JOURNAL ARTICLES}

$$
\text { (July } 1979 \text { - June 1980) }
$$

"Photoneutron Cross Sections for $55 \mathrm{Mn}$ and ${ }^{59} \mathrm{Co}, " \mathrm{R}$. A. A.lvarez, B. L. Berman, D. D. Faul, F. H. Lewis, Jr., and P. Meyer, Phys. Rev. C 20, 128 (1979)

"Determination of the Giant E2 Isoscalar Resonance for 236U," J.D.T. Arruda Neto, B. L. Berman, S. B. Herdade, and

I. C. Nascimento; Lett. Nuovo Cim. 26, 487 (1979)

"Alpha Decay Amplification in Superdeformed Nuclei: An Important New Mechanism of Nuclear De-excitation at High Angular Momenta," M. Blann, Phys. Lett. 88B, 5 (1979)

"Decay of Deformed and Superdeformed Nuclei Formed in Heavy Ion Reactions," M. Blann, Phys. Rev. C 21, 1770 (1980)

"Experimental Determination of Photofission Neutron Multiplicities for $235 \mathrm{U}, 236 \mathrm{U}, 238 \mathrm{U}$, and 232 Th Using Monoenergetic Photons," J. T. Caldwell, E. J. Dowdy, R. : A. Alvarez, B. L. Berman and P. Meyer, Nucl. Sci. Eng. 73,153 (1980)

"Giant Resonance for the Actinide Nuclei: Photoneutron and Photofission Cross Sections for $235 \mathrm{U}, 236 \mathrm{U}, 238 \mathrm{U}$, and $232 \mathrm{Th}, " \mathrm{~J}$. T. Caldwell, E. J. Dowdy, B. L. Berman, R. A. Alvarez and P. Meyer, Phys. Rev. C 21, 1215 (1980)

"Self-Shielded Fission Rates for Uranium-235," J. B. Czirr, Nucl. Sci. Eng. '70, 307 (1979).

"Pure Resonance Model for Radiative Capture of Fast Nucleons," F. S. Dietrich and A. K. Kerman, Phys. Rev. Lett. 43, 114 (1979)

"Photodisintegration of 3H," D. D. FauT, B. L. Berman, P. Meyer, and ก. L. Olson, Phys. Rev. 44, 129 (1980)

"A High-Pressure Thin-Window Gas Cel1," R. C. Haight, C. Rambo, J. Cormier and J. L. Garibaldi, Nucl. Inst. Meth. 164, 613 (1979)

"A Secondary-Electron Time Pickoff for Pulsed Charged-Particle Beams," R. C. Haight and D. R. Dalgas, Nucl. Inst. Meth. 165, 55 (1979)

"Neutron and Gamma-Ray Spectra from 232Th, 235u, 238 U, and 239Pu After Bombardment with 14-MeV Neutrons," L. F. Hansen, C. Wong, T. T. Komoto, B. A. Poh1, E. Goldberg, R. J. Howerton and W. M. Webster, Nucl. Sci. Eng. 72, 35 (1979)

"Least Squares Method for Analyzing Broadened X-Ray Line Shapes," R. H. Howell and D. L. Matthews, J. Quant. Spectrosc. Radiat. Transfer 23, 513 (1979) 
"( $\left.p, p^{\prime} \gamma\right)$ Spin-Flip Measurement for $1^{+}$States in ${ }^{12} C$ at $E_{p}=23.5-27 \mathrm{MeV}, "$ R. H. Howell, F. S. Dietrich, D. W. Heikkinen and F. Petrovich, Phys. Rev. C 21, 1153 (1980)

"Effect of the Tensor Force on Gamma-Ray De-excitation Angular Distributions from Dipole States Populated in the $12 c\left(p, p^{\prime}\right)$ Reaction," R. H. Howell, F. S. Dietrich and F. Petrovich, Phys. Rev. C 21, 1158 (1980)

"Positron Lifetime Study of $\mathrm{Fe}_{40} \mathrm{Ni}_{40} \mathrm{P}_{14} \mathrm{~B} 6$ Glass," R. H. Howell and R. W. Hopper, Scripta Metallurgica 13, 367 (1979)

"Photoneutron Reaction in 170: Ground-State Differential Cross Section at $98^{\circ}, "$ R. G. Johnson, B. L. Berman, K. G. McNeill, J. G. Woodworth and J.W. Jury, Phys. Rev. C 20, 27 (1979)

"Photoneutron Cross Sections for 170," J. W. Jury, B. L. Berman, D. D. Faul, P. Meyer, J. G. Woodworth, Phys. Rev. C 21, 503 (1980)

"Proton Inelastic Scattering at $12 \mathrm{MeV}$ on $154 \mathrm{Eu}, "$ R. G. Lanier, L. G. Mann, G. L. Struble, I. D. Proctor, and D. W. Heikkinen, Phys. Rev. C 20, 1279 (1979)

"Evidence for Nuclear Shape Coexistence at $N=88:{ }^{152} \mathrm{Eu}(\mathrm{d}, \mathrm{p})$ and $(d, t)$ Reactions," R. G. Lanier, G. L. Struble, L. G. Mann, I. C. Oelrich, I. D. Proctor, and D. W. Heikkinen, Phys. Rev. C 22, 51 (1980)

"Precise Measurement of the Charge Distribution Differences of the Oxygen Isotopes," H. Miska, B. Norum, M. V. Hynes, W. Bertozzi, S. Kowalski, F. N. Rad, C. P. Sargent, T. Sasanuma, and B. L. Berman, Phys. Lett. 83B, 165 (1979)

"Evidence for the Isovector Giant Quadrupole Resonance in 160 from the $160\left(\gamma, n_{0}\right) 150$ Reaction, "T. W. Phillips and R. G. Johnson, Phys. Rev. C 20, 1689 (1979)

"Evidence for Atomic Muon Capture by Fragments from Prompt Fission of Muonic $237 \mathrm{~Np}, 239 \mathrm{Pu}$, and 242Pu," W. U. Schröder, W. W. Wilcke, M. W. Johnson, D. Hilscher, J. R. Huizenga, J. C. Browne and D. G. Perry, Phys. Rev. Lett. 43,672 (1979)

"The Neutron Zero-Energy Total Cross Section and Scattering Lengths for Tritium," J. D. Seagrave, B. L. Berman and T. W. Phillips,

Phys. Lett. 91B, 200 (1980)

"Fusion of $58,60,62 \mathrm{Ni}$ with 113-170 MeV 40Ca Ions," B. Sikora, J. Bisplinghoff, W. Scobel, M. Beckerman, and M. Blann, Phys. Rev. C 20, 2219 (1979) 
PUBLICATION LIST - JOURNAL ARTICLES (Cont.)

"Comparisons of Experimental and Theoretical Nucleus-Nucleus Potentials for Heavy Ion Reactions," B. Sikora, M. Blann, W. Scobel, J. Bisplinghoff and M. Beckerman, Phys. Rev. C 21, 614 (1980)

"Observation of Channeling Radiation from Relativistic Electrons," R. L. Swent, R. H. Pantell, M. J. Alguard, B. L. Berman, S. D. Bloom, and S. Datz, Phys. Rev. Lett. $\underline{43}, 1723$ (1979)

"Actinide Muonic Atom Lifetimes Deduced from Muon-Induced Fission," W. W. Wilcke, M. W. Johnson, W. U. Schröder, D. Hilscher, J. R. Birkelund, J. R. Huizenga, J. C. Browne, and D. G. Perry, Phys. Rev. C 21, 2019 (1980)

" $(p, n)$ Reaction to Ground and Excited-State Analogs on the Samarium Isotopes: Importance of Two-Phonon Coupling Effects," C. Wong, V. R. Brown, V. A. Madsen and S. M. Grimes, Phys. Rev. C 20, 59 (1979)

"Power Broadening Effects in Resonant Scattering from Unstable Atomic Targets," S. D. Bloom, A. Goldberg and D. Tiszauer, Phys. Lett. 75A, 3 (1980) 
(July 1979 - June 1980)

"Channeling Radiation from Positrons," M. J. Alguard, R. L. Swent, R. H. Pantel 1, B. L. Berman, S. D. Bloom, and S. Datz; Proc. Eighth Particle Accelerator Conference, San Francisco, 1979;

IEEE Trans. Nucl. Sci. NS-26, 3865 (1979)

"Radiation from Channeled Leptons," M. J. Alguard, R. L. Swent, R. H. Pantel1, S. Datz, J. H. Barrett, B. L. Berman and S. D. Bloom, Proc. Eighth Int. Conf. Atomic Collisions in Solids, Hamilton, Canada, 1979; Nucl. Inst. Meth. 1.70, 7 (1980)

"Photodisintegration of $3 \mathrm{H}$ and $3 \mathrm{He}, " \mathrm{~B}$. L. Berman, D. D. Faul, P. Meyer and D. L. 0lson, Proc. Int. Conf. Nucl. Phys. with Electromagnetic Interactions, Mainz, Germany, 1979; Lecture Notes in Physics, Springer Verlag, Berlin, Vol. 108, p. 403 (1979)

"Decay of Deformed and Superdeformed Nuclei Formed in Heavy Ion Reactions,". M. Blann and T. T. Komoto, Proc. Twelfth Summer School on Nuclear Physics, Mikolajki, Poland, 1979; Nucleonika 25, 3 (1980)

"Linear Least-Square Analysis and Effective Interactions," S. D. Bloom, R. F. Hausman, and J. Larsen, Proc. Conf. $(p, n)$ Reaction and NucleonNucleon Force, Telluride, C0, 1979, Plenum Press, NY, p. 65 (1980)

"The Representative-Vector Method for Calculating Operator-Moments," S. D. Bloom and R. F. Hausman, Jr., Proc. Int. Conf. Theory and Application of Moment Methods in Many-Fermion Systems, Ames, IA, 1979, Plenum Press, NY, p. 151 (1980)

"Coherent Radiation from Correlated Collisions of Positrons with Si," S. Datz, J. H. Barrett, M. J. Alguard, R. L. Switt, R. H. Pantêll, B. L. Berman, and S. D. Bloom, Proc. Int. Conf. Physics of Electronic and Atomic Collisions, Kyoto, Japan, p. 976 (1979)

"A High Intensity Rotating Target $T(d, n)$ Neutron Source," J. C. Davis, Proc. Conf. High LET Radiations in Clinical Radiotherapy, Hague, The Netherlands, 1978, Plenum Press, NY, p. 132 (1979)

"RTNS-II Neutron Sources: Status Report," J. C. Davis, D. W. Heikkinen, J. L. Held, C. M. Logan and J. E. Osher, Proc. Eighth Particle Accelerator Conference, San Francisco, 1979; IEEE Trans. Nuc1. Sci. NS-26, 3058 (1979)

"Limits of Thermodynamic Models for Nuclear Level Densities," S. M. Grimes, Proc. Int. Conf. Theory and Application of Moment Methods in ManyFermion Systems, Ames, IA, 1979, Plenum Press, NY, p. 17 (1980) 
"Hauser-Feshbach Calculations of the Fission Neutron Spectrum," F. S. Dietrich and J. C. Browne, Proc. 25th Annual Mtg. Am. Nuc1. Soc., Atlanta, GA, 1979; Trans. ANS 32, 728 (1979)

"Calculation of Spin Cutoff Parameters Using Moment Techniques," S. M. Grimes, Int. Conf. on Theory and Applications of Moment Methods in Many Fermion Systems, Ames, IA, 1979, Plenum Press, NY, p. 273 (1980)

"Measurements and Calculations of the Neutron Spectra from Materials Used in Fusion-Fission Reactors," L. F. Hansen, R. J. Howerton, T. T. Komoto, B. A. Pohl and C. Wong, Proc. 25th Annual Mtg.

Am. Nuc1. Soc., Atlanta, GA, 1979; Trans. ANS 32, 663 (1979)

"A Positron Lifetime Study of Copper Irradiated by Energetic Protons or Energetic Neutrons," R. H. Howell, Proc. Fifth Int. Conf. on Positron Annihilation, Lake Tamanaka, Japan, p. 513 (1979)

"A Microprocessor-Controlled Portable Neutron Spectrometer,"

G. F. Hunt, R. C. Kaiffer, D. R. Slaughter, R. E. Strout II, and D. W. Rueppel, Proc. 26th Nuclear Science Symposium, San Francisco, 1979; IEEE Trans. Nuc1. Sci. NS-27, 757 (1980)

"Progress in Space Charge Lens Development," H. W. Lefevre and R. Booth, Proc. Eighth Particle Accelerator Conference, San Francisco, 1979; IEEE Trans. Nucl. Sci. NS-26, 3115 (1979)

"Energy Dependence of $V_{\tau}$ in the $(p, n)$ Reaction from 10-30 MeV," C. H. Poppe, Proc. Conf. $(p, n)$ Reaction and Nucleon-Nucleon Force, Telluride, CO, 1979, Plenum Press, NY, p. 461 (1980) 


\section{E-DIVISION SCIENTIFIC STAFF}

Permanent Staff

R. A. Alvarez

R. W. Bauer, Division Leader

J. A. Becker

B. L. Berman

H. M. Blann

S. D. Bloom

R. Booth

J. C. Browne (left July 1979)

H. S. Camarda (left August 1979)

D. D. Dietrich (started August 1979)

F. S. Dietrich (on research leave at Bruyeres-le-Chatel

R. J. Fortner January - Jul.y 1980)

S. M. Grimes (on research leave at Ohio University August 1978 - July 1979.)

R. C. Haight, Assoc. Div. Leader

L. F. Hansen

D. W. Heikkinen

R. E. Howe

R. H. Howel 1

T. T. Komoto

j. W. McClure (left March 1980)

P. Meyer

T. W. Phillips

W. L. Pickles (1eft February 1980)

B. A. Poht

C. H. Poppe, Assnr. Div. Leader

I. D. Proctor

D. R. Slaughter

R. M. White

C. Wong

J. G. Woodworth 
Visiting Faculty Member (Year)

J. D. Garcia, Arizona

Visiting Faculty Members (Surmer)

H. H. Barschall, Wisconsin

P. Chaplin, Lincoln

B. J. Dalton, Iowa State

J. W. Jury, Trent (Canada)

A. K. Kerman, MIT

H. W. Lefevre, Oregon

V. A. Madsen, Oregon State

F. L. Petrovich, Florida State

W. Scobel, Hamburg (Germany)

W. W. Smith, Connecticut 
Studying materials with positrons

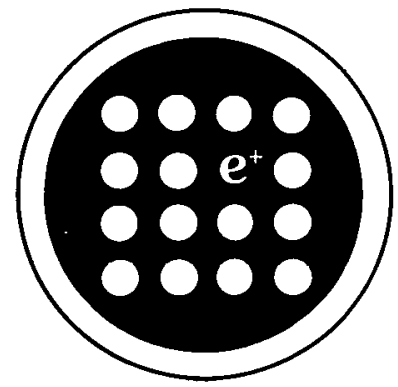

For further information contact Richard H. Howell (422-1977).

Information about the internal conditions of solids, including defect structure and phase changes, is needed in a variety of materials studies for weapons and fusion energy applications. We have developed a capability for determining solid-state characteristics with positron interactions, by measuring either positron lifetimes in the material or the Doppler broadening of the annihilation gamma rays. Two studies-one of an amorphous metallic alloy and another of radiation-induced damage in copper-demonstrate the effectiveness of such analysis.

Positron analysis of the internal structure of solids, including the defect structure at a microscopic level, is useful as a nondestructive testing technique. It is used in such diverse areas as the study of defects introduced by radiation or mechan. ical stress and the observation of phase changes in metals and alloys. Because of recent developments in the field of positron analysis, we can now obtain quantitative data that relate directly to the characteristics of materials, which in turn can depend on their test histories.

In a typical measurement, positrons from a radioactive source are introduced into a material. Once in the material, they slow doum and thermalize. In gases and in some insulators, the positron may then either encounter an electron in flight and be annihilated, or it may enter a temporary bourid state with an electron, forming a positronium atom similar to a hydrogen atom but with a positron rather than a proton nucleus.
Positronium does not occur in metals because of their high density of mobile electrons. Instead, nearby electrons at first screen the positron's positive charge. In a short time, one electron and the positron annihilate each other and produce two oppositely directed gamma rays, or photons, each with about $0.511-\mathrm{MeV}$ energy. The annihilation rate is a complicated function of the material's electronic structure and density, and the annihilation energy and angular correlation are determined by the relative momenta of the thermalized positron and the interacting electron.

Measuring the annihilation characteristics of positrons in a solid can give unique information about the environment around the 
positron at the time of its annihilation. Three qualities can be measured: the positron's lifetime, which is affected by the local electron density; the angular correlation of the two annihilation photons; and the energy spread introduced in the annihilation energy by Doppler broadening. Both positron lifetimes and Doppler broadening have been measured in experiments at LLL to study phase changes in amorphous metals, deformation characteristics of stainless steel, and radiation damage induced in copper and silver by protons from the LLL Cyclograaff or by $14-\mathrm{MeV}$ neutrons from the Rotating Target Neutron Source (RTNS I).

\section{Probing solids with positrons}

The introduction of a positron trapping model ${ }^{1}$ made possible the use of positrons in studying defects in solids. This model postulates that positrons are trapped at a defect site in a material at some rate. characteristic of the defect, of the material, and of the density of defects in the material. Once. trapped, the positron is annihilated when it combines with an electron near the trap. Such electrons typically exist in different densities and with energies different from those of the electrons in the conduction bands of the solid. Thus there are different annihilation rates and annihilation photon energies for trapped and free positrons.

Not all defect structures in solids are expected to trap positrons. For example, vacancies, vacancy clusters, and dislocations are all positron traps while interstitial atoms are not. Positron-lifetime or Doppler-broadening data may be analyzed by the trapping model to determine the trap parameters, the trap density, and associated quantities such as formation energies for the trap. These measurements have been made by other researchers on a variety of materials to study the formation energy of vacancy defects produced by thermal motion and to monitor the migration of defects 'through the various annealing stages. As a result, a large data base is available for comparison with new measurements.

\section{Measurement techniques}

Positron lifetimes are measured with two gamma-ray detectors designed to produce accurate timing information in their output. In the measurement, a radioactive positron source of sodium-22 is placed at the sample to be measured. This source simultaneously emits a gamma ray and a positron. One detector measures the prompt gamma ray; the second detector measures the annihilation gamma ray. The time difference between these two gamma-ray emissions is a measure of the positron's lifetime. We use two fast, plastic, scintillation detectors, developed at LLL, whose timing is coincident; with them we can accurately measure lifetimes of 100 ps. From the detection of a large number of annihilations, typically $10^{5}$, we obtain a spectrum of positron lifetimes characteristic of the sample. The lifetime or trapping-model parameters for each spectrum are then determined by computer analysis on a CDC 7600.

Doppler-broadening measurements, on the other hand, need no special source because only the annihilation gamma ray is detected.

The measurement simply requires a high-resolution, lithium-drifted germanium detector with stabilized electronics. The acquisition of Doppler-broadening data is straightforward; however, analysis of the data into trapping rates requires large data sets. To apply the trapping model, one compares the spectra obtained from samples with only a few traps and from samples with high enough trap densities for every positron to be trapped before annihilation. Because of this complexity and because the lifetime experiment provides more detailed information, we have emphasized positron lifetimes at LLL.

Figure 1 shows typical data for the positron-lifetime and Dopplerbroadening measurements of an annealed material with a low trap 


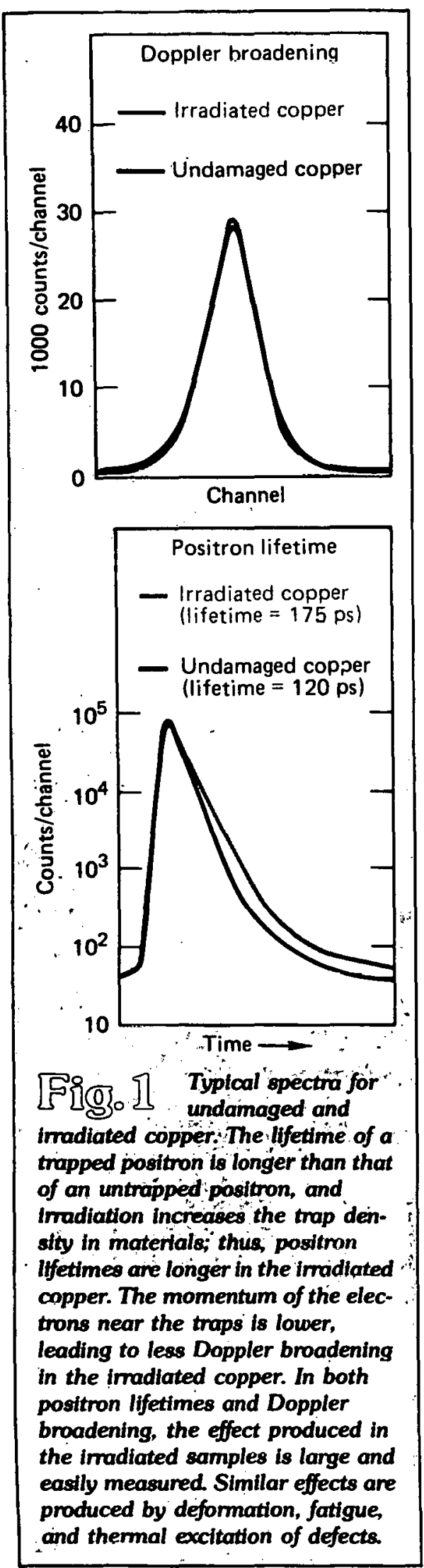

density and for the same material heavily damaged by irradiation. Damage generally serves to lengthen positron lifetimes because the positrons are trapped at defect sites when there is a lower electron density. At the same time, the electrons near a defect tend to have less momentum, leading to smaller Doppler shifts in the energy in the trapped positron.

\section{Alloy studies}

We have measured positron lifetimes in various amorphous metallic alloys ("metallic glasses") to better understand their internal structure and deformation process. Amorphous metallic alloys are new materials with potentially wideranging applications because of their extreme radiation resistance, high magnetic susceptibility, and overall strength. The microscopic details of these alloys are under investigation in many laboratories worldwide. Presently, the effects of deformation and radiation damage in the alloys are ill defined.

One of the alloys we have studied is $\mathrm{Fe}_{40} \mathrm{Ni}_{40} \mathrm{P}_{14} \mathrm{~B}_{6} \cdot{ }^{2}$ Positron lifetimes were measured in asreceived samples of the glassy alloy and in samples that were treated to deform, embrittle, or crystallize the alloy, thus changing its internal structure. The data obtained are listed in Table 1; three characteristics of these data are of particular interest.

First, positron lifetimes for the deformed and as-received samples are the same. The absence of any detectable change in these lifetimes suggests that no traps are formed in the deformation process. In contrast, crystalline alloys, when deformed, are known to have

\begin{tabular}{|c|c|}
\hline 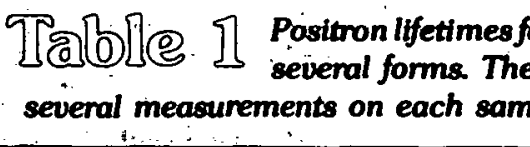 & $\begin{array}{l}{ }_{0} \mathrm{Ni}_{40} P_{14} \mathrm{~B}_{6} \text { prepared in } \\
\text { the standard deviation of }\end{array}$ \\
\hline Sample treatment & Positron lifetime, ps \\
\hline $\begin{array}{l}\text { As-received alloy } \\
\text { Deformied alloy } \\
\text { Alloy embrittled by } 300^{\circ} \mathrm{C} \text { anneal } \\
\text { Alloy embrittled by } 350^{\circ} \mathrm{C} \text { anneal } \\
\text { Crystallized alloy }\end{array}$ & $\begin{array}{l}158 \pm 2.5 \\
158 \pm 1.6 \\
156 \pm 1.6 \\
155 \pm 1.6 \\
152.5 \pm 0.8\end{array}$ \\
\hline
\end{tabular}


positron-trapping defects that increase the positron lifetime by as much as $50 \%$. Evidently, whatever defects deformation may introduce into the structure of amorphous alloys, they are either not much different from those in the virgin alloy or too few to affect positron annihilation characteristics. These results thus contradict many deformation models, which assume the generation of vacancies or stable dislocations.

Second, there is a small but significant change between the lifetime measured in the samples embrittled by annealing and the asreceived samples. Associated $x$-ray diffraction measurements indicate that the internal order of the embrittled samples is changed but still amorphous. These slight changes in phase are reflected in the small change in lifetime. Although these measurements show that the material remains amorphous with the annealing, there is a drastic change in the bulk mechanical properties: the material changes from being very springy to very brittle, even shattering on small impact.

Finally, the change in positron lifetimes between the alloy's glassy (as-received) phase and the crystalline phase is the same sign and about the same size as the change generally observed between a melt and solid phase in other materials. This similarity in lifetime behavior is consistent with the generally held assumpticn that the glassy phase is similar to the $\mathrm{ij}$ quid phase. In addition to probing amorphous-to-crystalline and liquid-to-crystalline changes, positron analysis is also suited to studying such phase conditions in solids as the crystalline phase, growth of Guinier-Preston zones (related to precipitation of solutes), long-range ordering in alloys, and martensitic transitions. ${ }^{3}$

\section{Comparative radiation- damage studies}

The radiation damage produced by $14-\mathrm{MeV} \mathrm{D}-\mathrm{T}$ fusion neutrons in the first wall and other components of a fusion reactor is an important problem in the development of such reactors. RTNS-II was constructed at LLL to produce $14-\mathrm{MeV}$ D-T neutrons for these studies. ${ }^{4}$ One question under study is the importance of the energies of the primary knock-on atoms (PKA) in determining radiation damage. As the energy of the PKA increases, the nuclear scattering decreases and a larger fraction of the atom's energy is lost to electrons. Also, the microscopic details of the defect change from isolated, randomly distributed point defects at low PKA energies to highly correlated, local clusters at higher PKA energies; this results in a complicated dependence of defects on PKA energy.

Positron annihilation measurements have been used at LLL to study the effects from irradiation at two sources: RTNS-I and the LLL Cyclograaff (a variable-energy proton source). ${ }^{5}$ The high-energy PKA spectra from these two sources are similar for protons with energies greater than $15 \mathrm{MeV}$; the lower energy PKA spectra produce significantly more damage in the proton case than in the neutron case. Thus a detailed comparison of the effects from irradiation with these two sources will illustrate similarities and differences in the energy dependence of the damage-producing mechanism and establish the relationship between long-term damage and theoretical values for instantaneous damage.

The focus of our radiationdamage studies was to investigate the dependence of long-term damage, or defect production, on PKA energy in room-temperature irradiations. Positron-lifetime measurements were used to probe defect densities and to identify defect characteristics. Commercially obtained, 99.999\%-pure copper was irradiated with neutrons to fluences as high as $5 \times 10^{16} \mathrm{n} / \mathrm{cm}^{2}$ and with protons to fluences as high as $10^{18} \mathrm{p} / \mathrm{cm}^{2}$. We irradiated all samples at near room temperature and measured positron 
lifetimes. We then determined lifetimes for the traps and bulk material and obtained a trapping rate from each lifetime measurement.

Figure 2 illustrates the dependence of the trapping rate on dose for neutrons and protons. The values for the traps produced in the two cases are nearly the same, indicating that there may not be major differences in the natures of the traps. The relationship of the trapping rate to unit dose suggests that neutrons and protons produce similar amounts of damage; this suggestion disagrees with the generally used simple displacement model, which includes the damage of all PKA above the threshold for producing isolated point defects (i.e., about $0.030 \mathrm{keV}$ ). This model predicts that the neutrons will produce about half the damage that protons do.

As shown in Fig. 3, the simple displacement model also does not describe the energy dependence of the long-term defect concentration observed in our experiments. The calculated rise in damage production at lower proton inadiation energies does not occur. The calculated increase in the damage production rate for irradiations with lower energy protons is mainly due to the increased probability of small-angle Coulomb scattering for these protons, and the damage produced is more widely distributed point defects rather than clusters. If defects produced with higher energy PKA are more stable, then the disagreement between the calculated and experimental data can be explained.

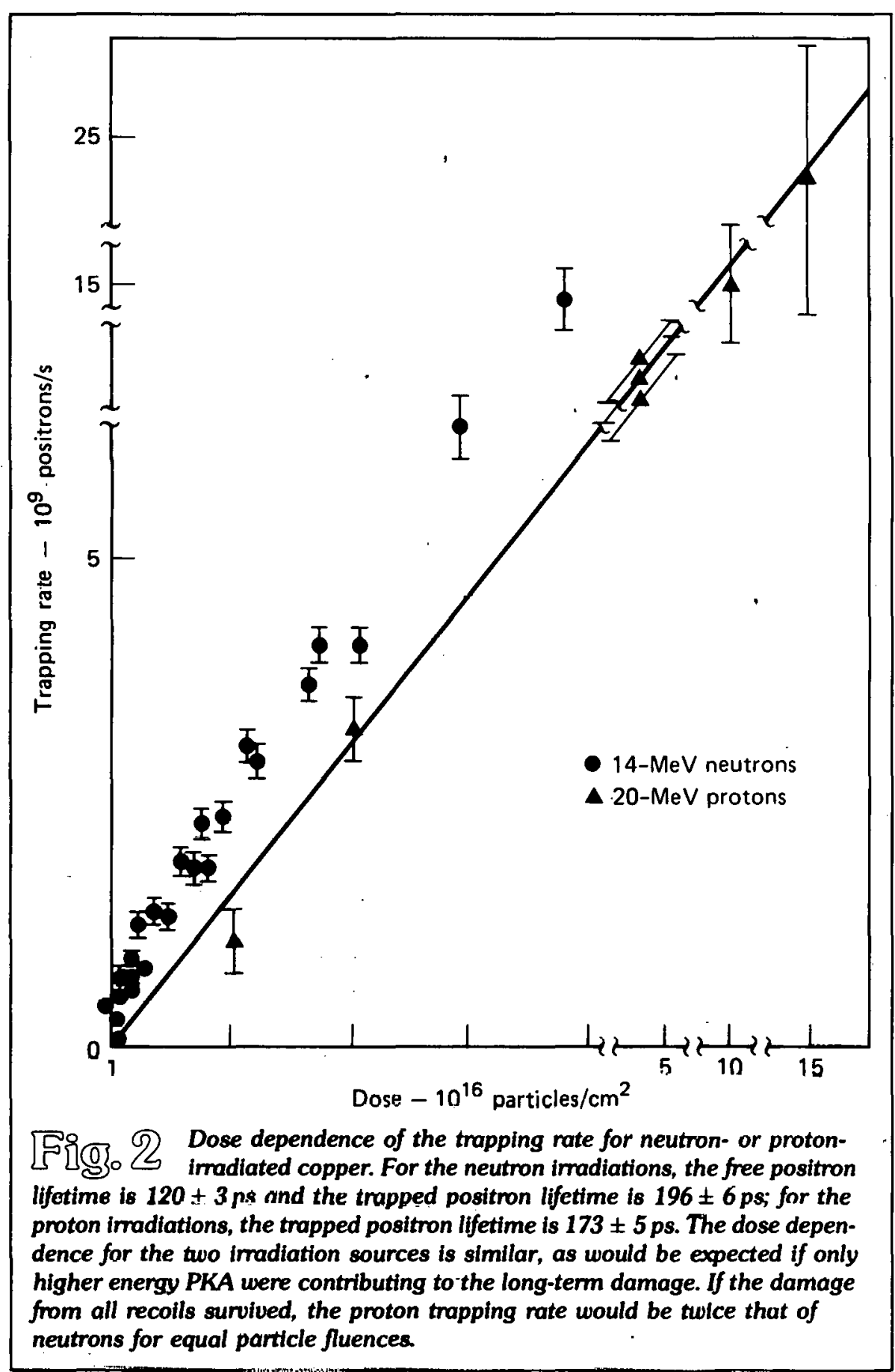


To try to understand the data, we made damage-production calculations with the displacement model for three much higher proton-energy thresholds: 20,55 , and $100 \mathrm{keV}$ (Fig. 3). These calculations are obtained by setting an energy cutoff in the PKA spec- trum and then calculating damage with the displacement model for all recoils above that cutoff. The $55 \cdot \mathrm{keV}$ threshold produces the best fit with data from our experiments. When we include this threshold in the comparison of our neutron and proton irradiations, the two data

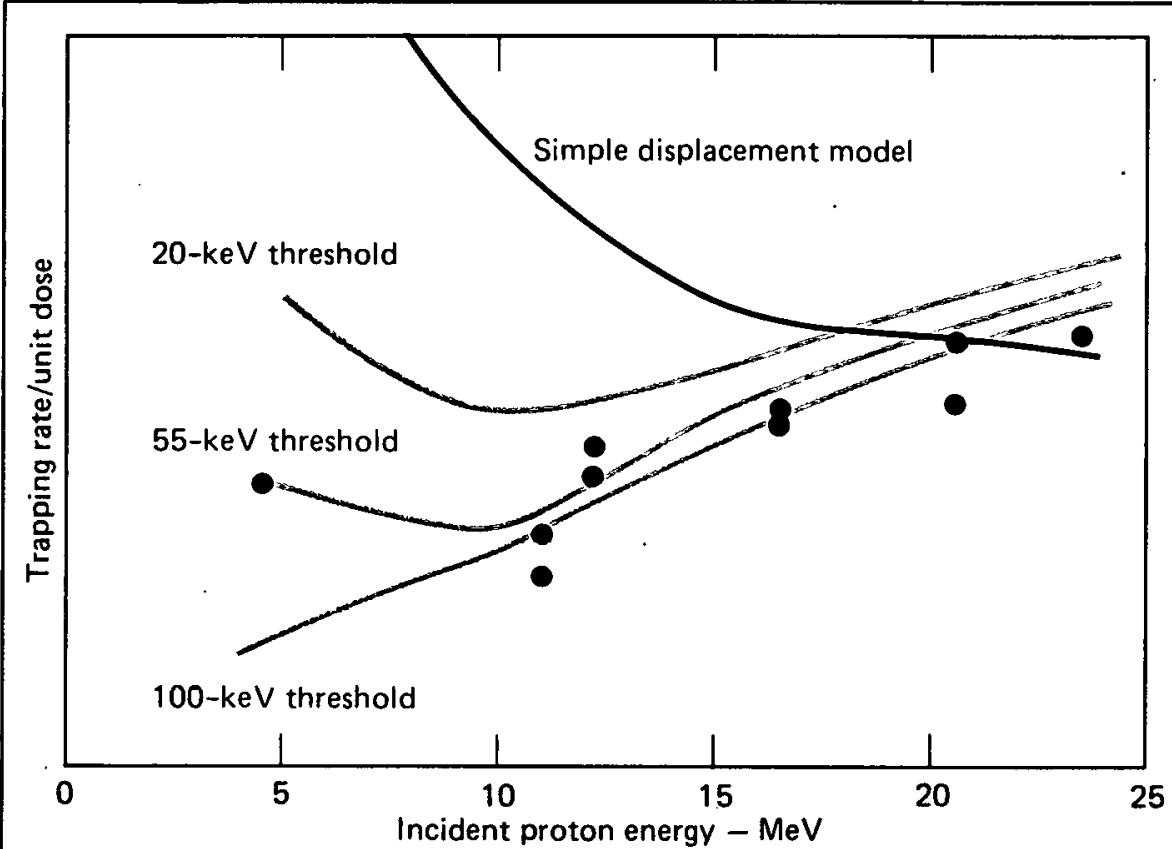

둥 की Dependence of trapping rate on incident proton energy. Since lower energy protons produce lower energy PKA in copper, these data are sensitive to the dependence of the long-term damage on the PKA energy spectrum. The simple displacement model does not describe the dependence of the defect production rate on proton irradiation energy found in our experiments. For this model to apply, it must include an energy threshold for the PKA spectrum. Calculation of the damage with such a threshold suggests that recoils below $55 \mathrm{keV}$ are not active in the production of long-term damage. This threshold is consistent with the dose-dependence data in Fig. 2.

sets then agree quantitatively within the accuracy of the damage calculations. The threshold model thus can be interpreted to show the relative importance of the highenergy PKA in damage production at room temperature.

\section{Future work}

We plan future investigations along the lines of the alloy and radiation-damage studies described above. We shall also study void formation and the energy threshold in the PKA spectrum for proton and neutron irradiations, as well as other radiation damage. New work in phase changes will include a detailed look at the liquid-melt boundary in pure metals.

Key words: annihilation reactions, Doppler broadening, nondestructive testing, photons-annihilation, PKA, positronium, pusitrons.

\section{Notes and references}

1. W. Brant, in Proc. Intem. Conf. Positron Annihilation, 1967 (Academic Press, New York, 1967), p. 80.

2. R. H. Howell and R. W. Hopper, "Positron Lifetime Study of $\mathrm{Fe}_{40} \mathrm{Ni}_{40} \mathrm{P}_{14} \mathrm{~B}_{6}$ Glass;" Scripta Met. 13, 367 (1979).

3. A description of current developments in positron measurements is contained in Proc. Intern. Conf. Positron Annihilation, 5th, Lake Yamanaka, Japan, 1979.

4. For a description of RTNS-II, see the March 1978 Energy and Technology Review (52000-78-3), p. 16

5. R. H. Howell, "A Positron Lifetime Study of Irradiation Effects in Copper Irradiated with Energetic Protons," Phys. Reu. B 18, 3015 (1978), and R. H. Howell, A Positron Lifetime Study of Copper Irradiated by Energetic Protons or Energetic Neutrons, Lawrence Livermore Laboratory, Rept. UCRL-82159 (1979), to be published in Proc. Intern. Conf. Positron Annihilation, 5th, Lake Yamanaka, Japan, 1979. 
Probing nuclear structure with improved electron-scattering techniques

Electron-scattering techniques can probe some of the most fundamental properties of nuclei, including the charge and magnetization distributions of nuclear ground states and the transition charge densities between the ground and excited states. Measurements of these properties now can be made with greater precision than was previously possible, thanks to the availability of MIT's Bates facility with its linac and energy-loss spectrometer. Thus an opportunity has been created for obtaining and exploiting much more accurate electron-scattering data.

In order to take advantage of this opportunity, LLL and MII have been conducting a series of collaborative experiments in which thin, precision-scattering targetsmanufactured using LLL's advanced chemical and metallurgical capabilities-are used in electronscattering measurements at the Bates facility. We have made such measurements on the important rare isotopes of oxygen (oxygen-17 and oxygen-18). We are extending these studies to the rare silicon isotopes and complementing them

We have been obtaining a new generation of electron-scattering data-of better precision and resolution than previously attainablein a collaborative program with MIT that began in 1976. The scatlering measurements are made with the linac and energy-loss spectrometer at MIT's Bates facility, using precision targets from $L L L$ made with our advanced chemical and metallurgical capabilities. We have obtained new results of elastic and inelastic scattering measurements on the rare isotopes of oxygen (oxygen-17 and oxygen-18). These results have yielded new insights into the electromagnetic structure of these nuclei and at the same time have opened new questions.

with proton-scattering studies at the Indiana University Cyclotron

Facility.

Because of the high energy resolution of the energy-loss spectrometer, we can use composite targets and isolate the three oxygen isotopes by the well-resolved peaks resulting from the differing recoil energies. The ratios of the various isotopic components can be measured with extremely good precision, and hence we can determine the ratios of form factors very accurately (to one part in a thousand). The form-factor ratios in turn can be translated into very precise information about the changes in the charge distribution that are induced by the addition of one or two nucleons to the "normal" oxygen-16 core (i.e., in going to oxygen-17 and oxygen-18).

Also of interest is the magnetization distribution of the oxygen 17 ground state, since oxygen-17 has the magnetic moment of a single free neutron. Backscattering elastic measurements have enabled us to evaluate existing models for predicting the magnetization distribution.

Besides the ground state, many other states are resolved, in the inelastic-scattering spectrum, which are relevant and of interest. In particular, the E2 transitions (as well as quadrupole moments) for oxygen18 indicate strong effective charges associated with the valence (orbital) neutrons.

Thin targets of $\mathrm{BeO}$, in which the oxygen was enriched in oxygen-17 or oxygen-18, were manufactured at $\mathrm{LLL}$ from $\mathrm{H}_{2} \mathrm{O}$ samples. ${ }^{1}$ We began obtaining electron-scattering data on these targets at the Bates facility in 1976. More recently, we have synthesized $\mathrm{BeO}$ targets from oxygen gas highly enriched (about 90\%) in oxygen-17 and have obtained electron-scattering data on them as well. These latter data are especially valuable for low momenturn transfers, where interference from charge scattering from the 
even oxygen isotopes is a serious problem.

Ground-state charge distributions-axygen-17 and oxygen-18. Our first goal was to understand the problems associated with the comparison of elastic scattering of electrons (at $90 \mathrm{deg}$ ) from the ground-state charge distributions of oxygen-16, oxygen-17, and oxygen-18. This work has been completed, and a detailed, modelindependent analysis of the data has been made. ${ }^{2}$ As a result, we have measured the ratios of these cross sections much more accurately than ever before; from these improved data we have determined the charge-distribution differences between oxygen-17,18 and oxygen-16.

These results are shown in Fig. 1 , where the charge density $\rho$ is plotted as a function of the distance $r$ from the center of the nucleus for oxygen-16 and oxygen-18; on this

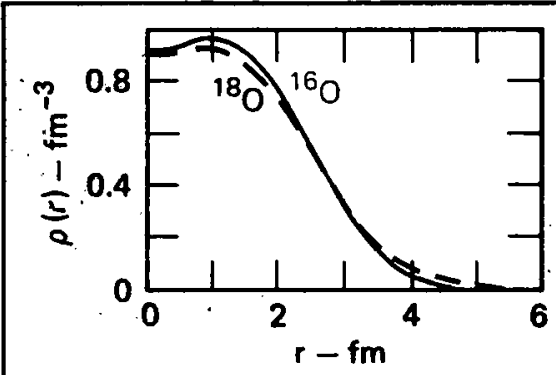

[SR 17 The nuclear groundSLGJo state charge distribution $\rho(\mathrm{r}$ ) for axygen-16 (solid line) and for axygen-18 (dashed line). On this scale, $\rho(r)$ for axygen17 would be so dose to that for axygen-16 as to be indistinguish- . able from it. scale, $\rho(r)$ for oxygen-17 would be indistinguishable from that for oxygen-16. In fact, the difference between the root-mean-square radii of oxygen-17 and oxygen-16 is only about one-tenth of that between oxygen-18 and oxygen-16 $(-0.008 \pm 0.007 \mathrm{fm}$ as compared to $+0.074 \pm 0.005 \mathrm{fm}$ ). Thus, it appears that the addition of a single valence neutron to the oxygen-16 core perturbs the charge distribution of the core hardly at all, while the addition of a pair of valence neutrons perturbs the core greatly. This result was foreshadowed by our earlier photonuclear work at $\mathrm{LLL}^{3}$ and appears to be confirmed by our recent proton-scattering measurements at Indiana. ${ }^{4}$

Ground-state magnetization distribution-oxygen-17. Our second goal was to map the magnetization distribution for oxygen 17 by making backscattering measurements. For the first phase of this project we took data scattered at $160 \mathrm{deg}$ from a target enriched to only $20 \%$ in the oxygen-17 isotope. The additional data necessary at lower

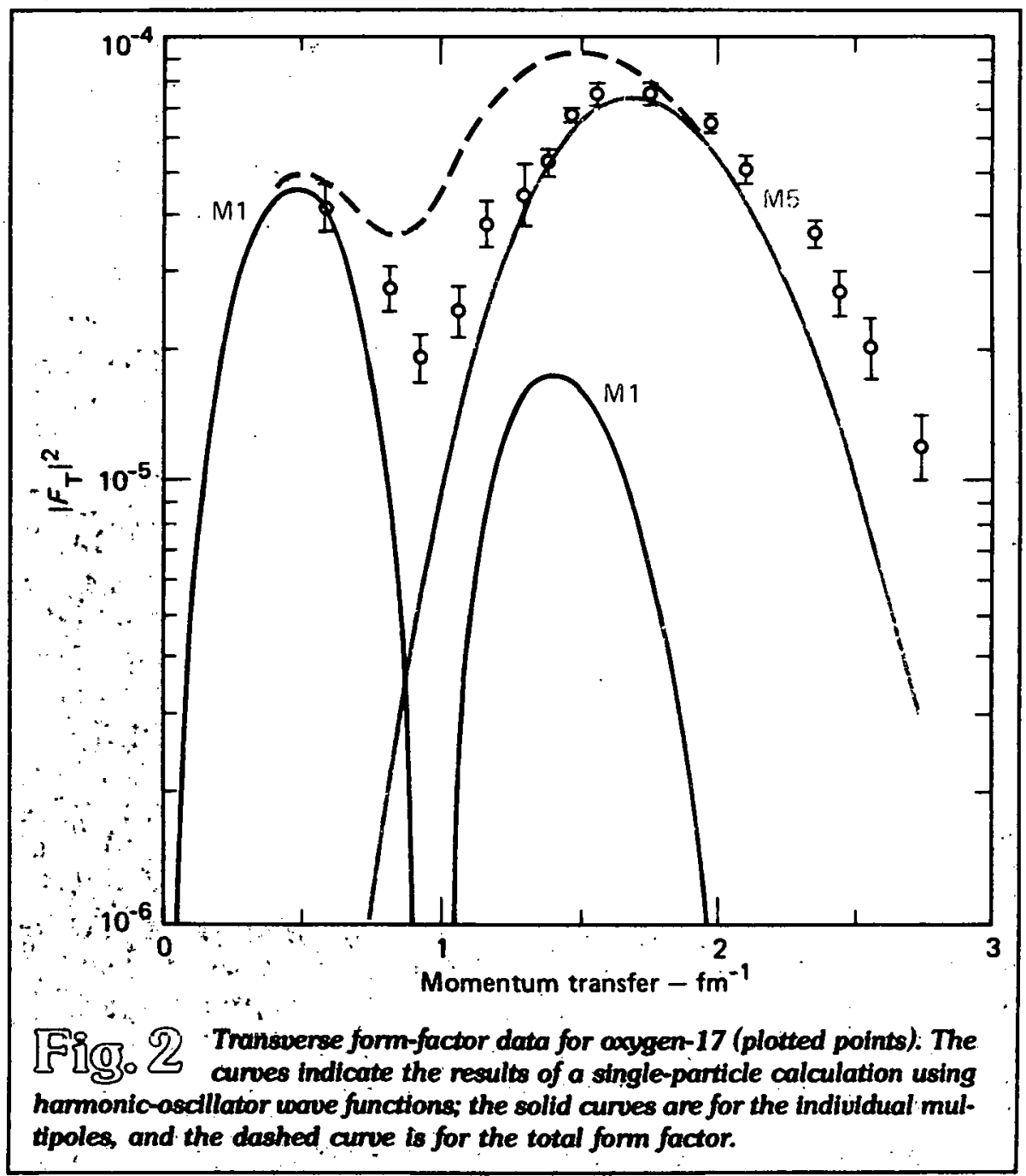


momentum transfers were obtained with the new 180-deg scattering system at Bates, using targets having a much higher enrichment (about 85\%) of oxygen-17.

The results of this work ${ }^{5}$ are shown in Fig. 2, where the square of the transverse form factor $F_{\mathrm{T}}$ (the Fourier transform of the magnetization distribution) is plotted as a function of the momentum transferred to the nucleus by the bombarding electron. Since the ground-state spin of oxygen-17 is $5 / 2$, three transitions are possible: magnetic dipole (M1), octupole
(M3), and $2^{5}$-pole (M5). The solid curves show the single-particlemodel predictions for these three components, and the dashed curve shows their sum. The data deviate significantly from the prediction of this simple model. Moreover, we find that even much more sophisticated models cannot account for these deviations, thus showing that more theoretical effort is needed on a subject heretofore thought to be well understood.

Excited states of oxygen-18. A problem we were able to study for

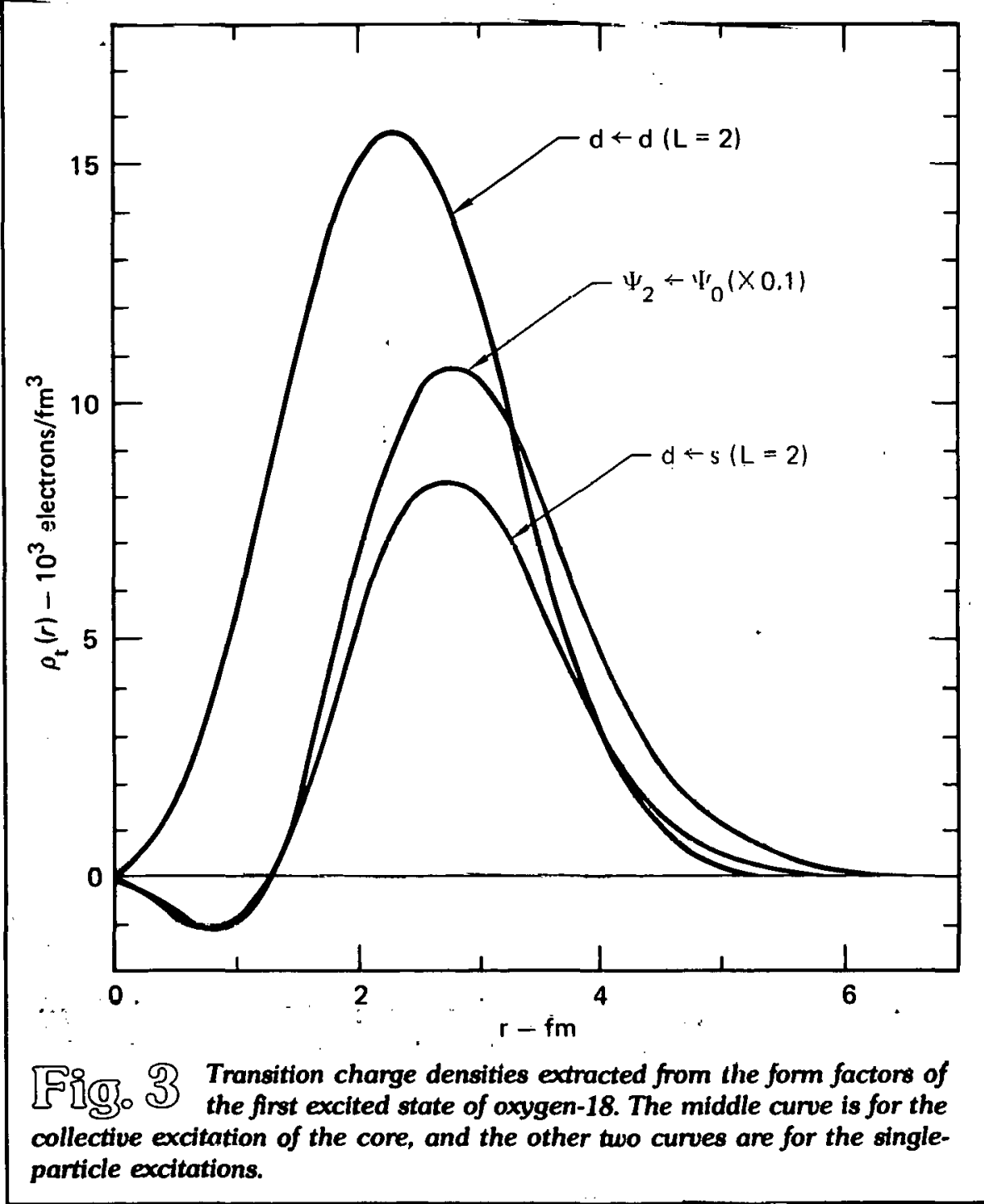

the first time-because of our highresolution measurement technique-is the excitation of oxygen18 to states in the energy region below $8 \mathrm{MeV}$. We have observed the excitation of eight even-spin, positive-parity states in this region and have measured the form factor for each state through a wide range of momentum transfer. A detailed analysis of these data has been made using a hybrid model in which these states are described by linear combinations of two-particle configurations and collective excitations of the core. ${ }^{G}$

A result of this work, for the excitation of the lowest lying $2^{+}$state, is shown in Fig. 3, where the transltion charge density $\rho_{\mathrm{t}}$ is plotted as a function of $r$. We see that the collective component of the nuclear excitation (the middle curve in Fig. 3) has nearly the same spatial distribution as the s-state-to$\mathrm{d}$-state single-particle component (lower curve), rather than the d-to$\mathrm{d}$ component (upper curve)-a direct indication that collective excitation of oxygen-18 takes place preferentially near the nuclear surface, as is expected theoretically. This result, when combined with the other results of these measure ments, indicates that this kind of hybrid model might very well provide a useful framework within which to understand the electromagnetic structure of such nuclei. 
Rare silicon isotopes. We plan shortly to begin electronscattering measurements on the rare isotopes of silicon, silicon-29 and silicon-30, using thin $\mathrm{SiO}_{2}$ targets. These measurements will explore the effects of adding one or two valence neutrons to a core (silicon-28) which, unlike oxygen16 , is thought to be strongly deformed. Recently, in a collaboration between LLL and the University of Melboume, a related series of photonuclear measurements has been begun as well.

For further information contact Barry L. Berman (422-0106).

Key words: charge distribution; electron scat tering-measurements; excited states; magnetization distribution; oxygen isotopes $0-16$; oxygen isotopes $\mathrm{O} \cdot 17$; oxygen isotopes $0 \cdot 18$.

\section{References}

1. R. H. Condit, Lawrence Livermore Laboratory, private communication (1979).

2. H. Miska, B. Norum, M. V. Hynes, W. Bertozzi, S. Kowalski, F. N. Rad, C. P. Sargent, T. Sasanuma, and B. L. Berman, "Precise Measurement of the Charge Distribution Differences of the Oxygen Isotopes," Phys. Lett. 83B, 165 (1979).

3. B. L. Berman, D. D. Faul, R. A. Alvarez, and P. Meyer, "Photoproton Cross Section for ${ }^{18} \mathrm{O}$ as a Measure of the Effect of the Valence Neutrons on the ${ }^{16} \mathrm{O}$ Core," Phys. Reu. Lett. 36, 1441 (1976).

4. B. Norum, W. Bertozzi, M. Deady, W. Hersman, M. Hynes, J. Kelly, H. Miska, F. Rad, K. Amos, A. Bacher, G. Baranko, G. Emery, C. Foster, W. Jones, D. Miller, A. Picklesimer, G. Walker, D. Weiss, and B. Berman, "Intermediate Energy Proton Scattening from Oxygen 16,17, 18," Bull. Amer. Phys. Soc. 23, 960 (1978).

5. M. V. Hynes, H. Miska, B. Norum, W. Bertozzi, S. Kowalski, F. N. Rad, C. P. Sargent, T. Sasanuma, W. Turchinetz, and B. L. Berman, "Electron Scattering from the GroundState Magnetization Distribution of ${ }^{17} \mathrm{O}$," Phys. Reu. Lett. 42, 1414 (1979).

6. B. E. Norum, M. V. Hynes, H. Miska, W. Bertozzi, S. Kowalski, F. N. Rad, C. P. Sargent, T. Sasanuma, W. Turchinetz, and B. L. Berman, "Inelastic Electron Scattering from

${ }^{18} \mathrm{O}$," to be submitted to Phys Reu. C(1979). 


\section{Observation of channeling radiation}

The phenomenon of the channeling of positively charged ions in preferred axial or planar directions in single crystals has been known for about twenty years. Particles moving along a crystal axis should have a corkscrewlike motion; those moving parallel to crystal planes should oscillate between the trapping planes with a transverse motion close to that of a harmonic oscillator. At relativistic energies, either motion should lead to the emission of narrow-band radiation that is strongly peaked forward, i.e., in the direction of the chargedparticle beam. The existence of channeling radiation and some of its properties were predicted recently by both Russian and American physicists.

In collaboration with the Department of Electrical Engineering at Stanford University and with Oak Ridge National Laboratory, we have observed radiation from channeled particles for the first time with a positron beam from the LLL electron-positron linac [see $M$. Alguard et al., Phys. Rev. Lett. 42, 1148 (1979)]. The observation was based on theoretical work at Stanford and on earlier positron and electron channeling and radiation experiments done at the LLL linac with thin silicon crystals. The latest results, also with thin silicon crystals, clearly show the structure in the radiation spectra that results from the channeling of $56-\mathrm{MeV}$ positrons (Fig. 2).

The top three plots in Fig. 2 show the spectra that result from channeling along the three major crystal planes; the fourth plot shows the spectrum from channeling along the $\langle 110\rangle$ axial direction. All curves have been normalized to the bremsstrahlung spectrum (i.e., the spectrum characterizing "unoriented" directions of the crystal with respect to the beam direction). Accordingly, the ordinate units represent spectral ratios rather than intensities. The peak energies for the

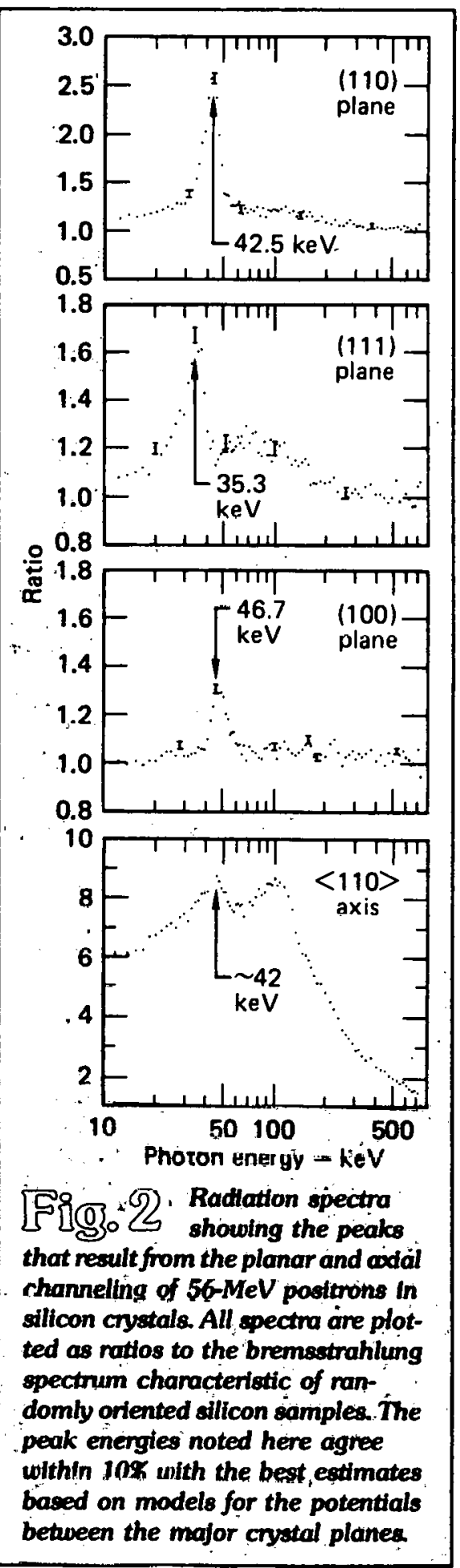

curves agree within $10 \%$ with the best estimates based on models for the potentials between major crystal planes. The radiation spectrum from the axial direction also shows evidence of planar channeling in its $42 \cdot \mathrm{keV}$ peak, since this peak is contained in the (110) plane. The peak energies are given approximately by the expression $2 \gamma^{3 / 2} \epsilon_{0}$, where $\gamma$ is the ratio of the energy of the positron to its rest mass and $\epsilon_{0}$ is the interplanar oscillator energy interval.

Future work at the linac will extend these measurements to other energies, angles, crystal species and thicknesses, and, perhaps most importantly, to the use of incident beams of electrons. Our long-range goal is to develop an intense, tunable, narrow-band, highly directional, and polarized source of $x$ rays. Such a source would be useful both for fundamental research and for a wide range of applications.

For further information contact Stewart D. Bloom (422.4519) or Barry L. Berman (422-9657). 


\section{Measuring the hydrogen and helium produced by fusion neutron reactions}

Hydrogen and helium produced by the reactions of fusion neutrons can significantly degrade the properties of structural materials used in future fusion reactors. We have recently measured the probability of forming hydrogen and helium in seven major elements and in two stainless-steel alloys. We have also investigated 11 isotopes of the elements to validate nuclear reaction model codes, which are essential in providing much of the neutronic data for fusion applications. We will complete studies of other elements of structural materials and will expand our investigation to include pertinent nonstructural elements. The resulting data base of cross sections for hydrogen and helium production will help us interpret complex radiation damage experiments.

radiation damage, their effects will likely be different in ways not yet well understood. To design and interpret radiation damage experiments, one must know how much hydrogen and helium are produced by transmutation.

We are measuring the probability of forming hydrogen and helium when fusion neutrons interact with various structural materials likely to be used in fusion reactors. Our method is to measure the charged particles (protons, deuterons, and alpha particles) from reactions induced by 14 - to $15-\mathrm{MeV}$ neutrons. ${ }^{1}$ In a bulk material, these charged particles stop after penetrating less than than $2 \mathrm{~mm}$; by electron attachment, the protons and deuterons then become hydrogen and the alpha particles become helium. With our magnetic quadrupole spectrometer, developed in collaboration with researchers at the University of Wisconsin, we can measure the charged particles much more cleanly than with previous techniques. Once we have those measurements, we can integrate the spectra over the energy and the angular distribution to obtain a total production cross section.

Experimental results. In our work to date, we have concentrated on the elements of such potential structural materials as most stainless steels (chromium, iron, nickel), some refractory metals (vanadium, niobium), and other commonly used metals (aluminum, titanium, copper). To check consistency and to test for the effect of impurities, we also measured charged-particle production in two stainless-steel alloys, SS-316 and SS-304.

The results, shown in Fig. 1, illustrate the wide range of probabilities of the reactions, quantified as cross sections, for these elements and alloys. Although the response of the structural materials to these gases likely will be dif- . ferent, we know from these data that the amount of hydrogen and helium produced by transmutation depends strongly on the constituents of the materials. For exam. ple, a factor-of-15 difference in hydrogen production was observed between niobium and nickel. 
Our data were taken at $15 \cdot \mathrm{MeV}$ neutron energy, slightly higher than the $14-\mathrm{MeV}$ fusion energy but experimentally much more convenient. To extrapolate the data to $14 \mathrm{MeV}$, nuclear reaction model codes are essential. ${ }^{2}$ To verify the accuracy of these codes and to understand the nuclear processes, we have measured charged-particle production in many of the stable isotopes of the elements studied: aluminum-27, titanium-46, titanium -48 , vanadium -51 , chromium-50, chromium-52, iron54, iron-56, nickel-58, nickel-60, copper-63, copper-65, and niobium-93. The predictions of the best model calculations are generally quite close to our measured cross sections.

A more stringent test of the models is if they can also reproduce the energy spectrum of the charged particles as well as the spectrumintegrated cross section. A favorable result is shown in Fig. 2, where the data are well described by a combination of two reaction models, one treating protons evaporated from the compound nucleus, the other describing the emission of protons before the nucleus comes to thermal equilibrium.

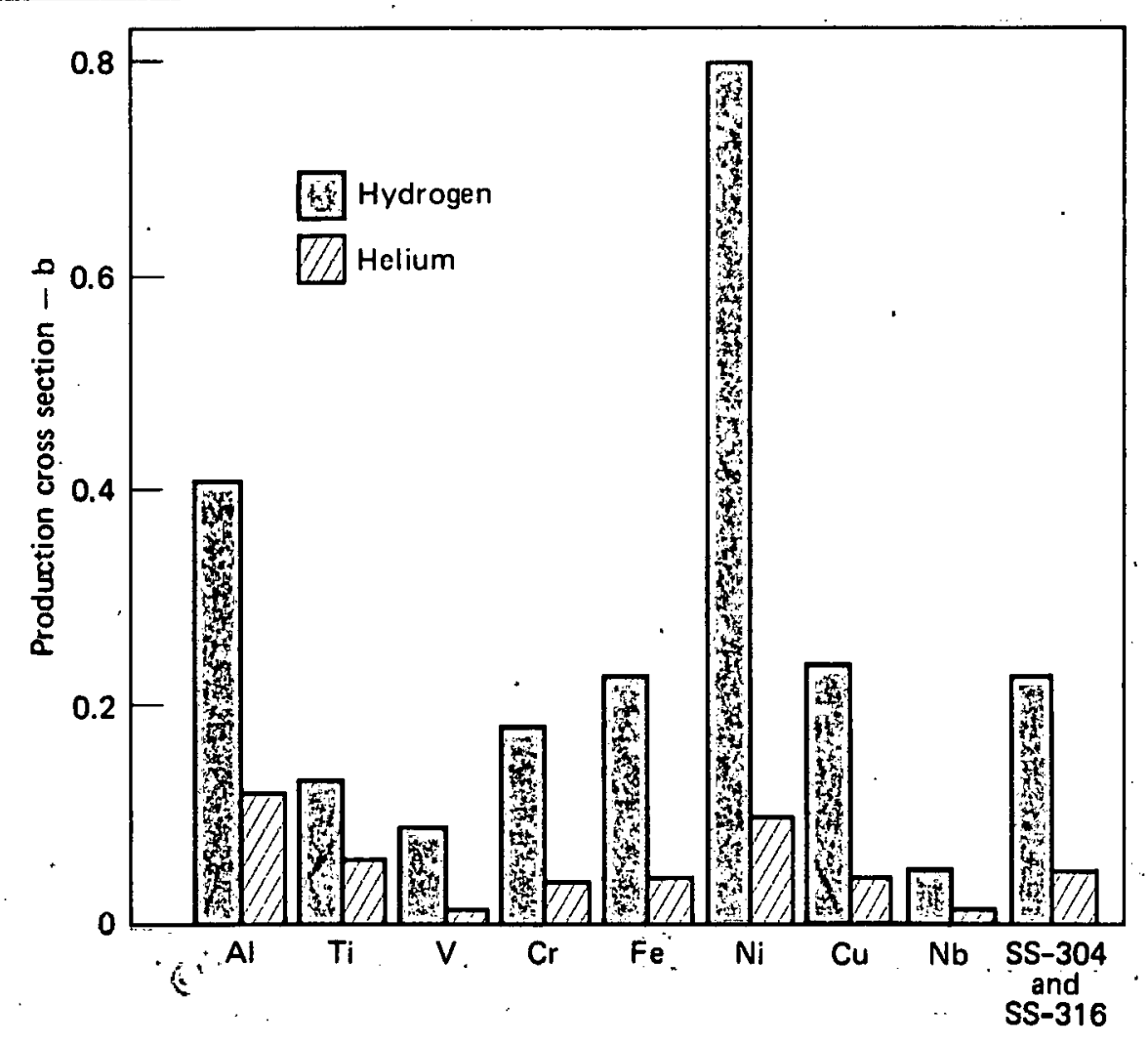

[Co] Production cross sections of hydrogen and hellum for 15-MeV SGSO neutrons on various elements and alloys proposed as structural materials for fusion reactors. Although the production cross sections for helium are smaller than those for hydrogen, the effects of helium on structural materials may be greater.

Our experimental data are being used by researchers at the Oak Ridge National Laboratory, the Los Alamos Scientific Laboratory, and the Hanford Engineering Development Laboratory, as well as by us, to check nuclear reaction model codes. The general result is that, for the elements and alloys studied, the codes describe the protonemission data quite well if reasonable parameters are used in

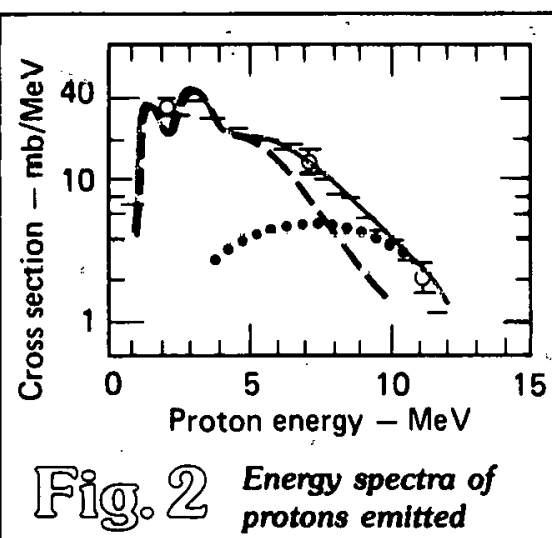

from chromium-52 when it is bombarded with 15-MeVneutrons. The horizontal bars denote the binned experimental data. The curves show the results of nuclearreaction-model calculations: the dashed curve represents a particle evaporation model; the dotted curve is for pre-equilibrium proton emission; and the solld curve shows the sum of the two calculations, in good agreement with the experimental data. 
the calculations. The alpha-particleemission data are described almost as well, but the deuteron data are very difficult to calculate. Fortunately, for reactor applications, the deuteron emission cross sections are small compared with those of protons.

Future work. Some elements of possible structural materials remain to be studied, such as molybdenum and zirconium, and are under investigation now. Nonstructural elements-carbon, lithium, boron, and oxygen - will be the next major area for investigation; all have likely applications in fusion reactors. Studying these elements differs from studying the heavier ones in that nuclear models probably will not describe the data accurately. The measurements must therefore be made at $14 \mathrm{MeV}$, the neutron energy of fusion reactors. The Rotating Target Neutron Source (RTNS-I) at LLL has been modified to permit these measurements.

With this rapidly expanding data base of cross sections from hydrogen and helium production, we will have more confidence in interpreting the complex radiation damage experiments soon to be undertaken at RTNS-II (the Laboratory's new irradiation facility) $)^{3}$ and at other irradiation test facilities.
For further information contact Robert C. Haight (422-4510).

Key words: charged-particle detectors; fast neutrons-cross sections; fusion reactors; materials-effects of radiation; spectrometerquadrupole.

\section{Notes and references}

1. The initial development and application of the magnetic quadrupole spectrometer are described in the April 1977 Energy and Technology Review (UCRL-52000-77-4), p. 14.

2. Some LLL activities in nuclear modeling are described in the May 1979 Energy and Technology Review (UCRL-52000-79-5), p. 1.

3. RTNS-I and RTNS-II are described in the March 1978 Energy and Technology Review (UCRL-52000-78-3), p. 16. 
This report was prepared as an account of work sponsored by the United States Government. Neit her the United States nor the United States Department of Energy, nor any of their employees, nor any of their contractors, subcont ractors, or their employees, makes any warranty, express or implied, or assumes any legal liability or responsibility for the accuracy, completeness or usefulness of any information, apparatus, product or process disclosed, or represents that its use would not infringe privately owned rights.

Reference to a company or product name does not imply approval or recommendation of the product by the University of California or the U.S. Department of Energy to the exclusion of others that may be suitable. 
Technical Information Department $\cdot$ Lawrence Livermore Laboratory

University of California - Livermore, California 94550

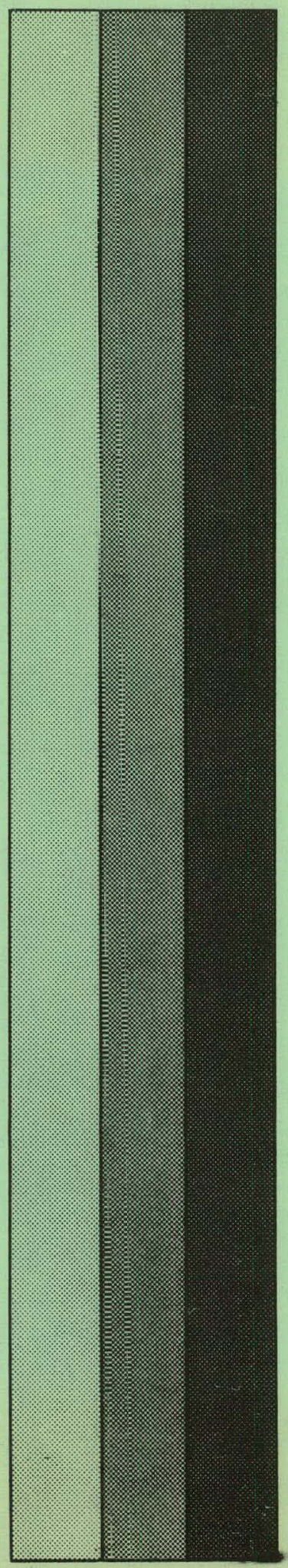

